\title{
$1 \quad$ Loss of Cohesin regulator PDS5A reveals repressive role of Polycomb loops
}

3 Daniel Bsteh"1,2,4, Hagar F. Moussa², Georg Michlits², Ramesh Yelagandula², Jingkui

4 Wang ${ }^{3}$, Ulrich Elling ${ }^{2}$, Oliver Bell ${ }^{1,2,5,{ }^{*}}$

5

$6{ }^{1}$ Department of Biochemistry and Molecular Medicine, Norris Comprehensive Cancer

7 Center, Keck School of Medicine, University of Southern California, Los Angeles, CA, 8 USA.

$9{ }^{2}$ Institute of Molecular Biotechnology of the Austrian Academy of Sciences (IMBA), 10 Vienna BioCenter (VBC), Vienna, Austria

$11{ }^{3}$ Research Institute of Molecular Pathology (IMP), Vienna BioCenter (VBC), Vienna,

12 Austria

$13{ }^{4}$ Vienna BioCenter PhD Program, Doctoral School of the University of Vienna and

14 Medical University of Vienna, Vienna, Austria

$15{ }^{5}$ Lead contact

16 * Correspondence: oliver.bell@med.usc.edu (O.B.) 


\section{ABSTRACT (146 words)}

Polycomb Repressive Complexes 1 and 2 (PRC1, PRC2) are conserved

19 epigenetic regulators that promote transcriptional silencing. PRC1 and PRC2 converge

20 on shared targets, catalyzing repressive histone modifications. In addition, a subset of

$21 \mathrm{PRC1/PRC2}$ targets engage in long-range interactions whose functions in gene silencing

22 are poorly understood. Using a CRISPR screen in mouse embryonic stem cells, we

23 identified that the cohesin regulator PDS5A links transcriptional silencing by Polycomb

24 and 3D genome organization. PDS5A deletion impairs cohesin unloading and results in

25 derepression of subset of endogenous PRC1/PRC2 target genes. Importantly,

26 derepression is not associated with loss of repressive Polycomb chromatin

27 modifications. Instead, loss of PDS5A leads to aberrant cohesin activity, ectopic

28 insulation sites and specific reduction of ultra-long Polycomb loops. We infer that these

29 loops are important for robust silencing at a subset of Polycomb target genes and that

30 maintenance of cohesin-dependent genome architecture is critical for Polycomb

31 regulation. 


\section{INTRODUCTION}

In metazoans, precise epigenetic regulation of gene expression enables the development of diverse cell types despite the same underlying genomic blueprint. Gene expression is primarily controlled by DNA-binding transcription factors directing the transcriptional apparatus. However, epigenetic mechanisms modulate chromatin to directly and indirectly regulate transcription. Polycomb repressive complexes are chromatin-modifiers and serve as prototypes of epigenetic gene regulation via histone modifications. Decades of research have cemented their roles in establishing and maintaining cell identity throughout development across organisms, ranging from Drosophila melanogaster to vertebrae ${ }^{1,2}$. Moreover, aberrant activity of Polycomb complexes and other epigenetic regulators contribute to diverse diseases, including cancer initiation and metastasis, highlighting the importance of understanding the pathological mechanisms ${ }^{3-10}$.

Polycomb group (PcG) proteins are conventionally grouped into Polycomb as their catalytic core subunit, which deposits H2AK119ub at its targets, whereas PRC2 contains the histone methyltransferase EZH1/2, which catalyzes H3K27me3 at targets 11-14. In vertebrates, PRC1 complexes have diversified into distinct subcomplexes based on incorporation of one of six paralogous PCGF proteins (PCGF1-6). PCGF2 or PCGF4 dictate assembly of canonical PRC1 (cPRC1) which specifically incorporates CBX

52 (chromobox-containing protein) subunits. CBX subunits endow CPRC1 with the capacity to bind H3K27me3, which promotes cPRC1 recruitment to PRC2 target genes and transcriptional silencing ${ }^{11,15,16}$. PCGF1, 3, 5 and 6 form variant PRC1 (vPRC1) complexes which harbor RING1 and YY1- binding protein (RYBP), or its paralogue YAF2 instead of CBX, and rely on PRC2-independent mechanisms of chromatin targeting. Thus, PRC1 and PRC2 are generally considered to exert their repressive functions in a synergistic manner, but they have different mechanisms of targeting, signaling and repression 59 (reviewed in ${ }^{17}$ ). 
Recent studies have established that VPRC1 can act upstream of PRC2 and cPRC1, and that its H2AK119ub deposition is critical for Polycomb-dependent gene

62 silencing ${ }^{18-20}$. Indeed, loss of PRC2 or cPRC1 does not substantially compromise the 63 repression of Polycomb target genes in mouse embryonic stem cells (mESCs) 64 expressing VPRC1 18,19. These findings have propelled VPRC1 to the center of 65 coordinating and establishing a repressive Polycomb chromatin domain. Although cPRC1 contributes minimally to H2AK119ub deposition, it possesses the unique capacity to mediate long-range 3D interactions between Polycomb target genes ${ }^{12,21-27}$, which has been shown to contribute to gene silencing in flies ${ }^{28}$.

The redundant functions of $\mathrm{VPRC} 1$ and $\mathrm{CPRC} 1$ complicate dissecting their individual mechanisms by genetic analysis ${ }^{18,19,29,30}$. To circumvent this limitation, we previously developed a Polycomb in vivo Assay that reports the activity of distinct PRC1 complexes. Briefly, we generated mESCs that can recruit ectopic cPRC1 or vPRC1 to an integrated TetO repeat flanked by fluorescent reporters (Fig. 1a) ${ }^{31}$. For instance, ectopic expression of a CBX7-Tet repressor domain (TetR-CBX7) fusion triggers the assembly of $\mathrm{cPRC} 1$ at the TetO sites, Polycomb-dependent histone modifications and reporter gene silencing. Binding of the TetR fusion is released upon addition of Doxycycline (Dox), and we found that more than $70 \%$ of cells maintained cPRC1induced, but not vPRC1-induced, silencing in the presence of Dox. We showed that sequence-independent propagation of cPRC1-induced silencing requires H3K27me3 and H2AK119ub, suggesting that it relies on PRC1/PRC2 feedback. However, the mechanism and players required for heritable PRC1/PRC2-mediated silencing, and whether they are the same at all target genes, remain incompletely understood.

Here we performed a CRISPR-mutagenesis screen to identify novel regulators of

85 for repression of canonical Polycomb target genes. Unexpectedly, loss of PDS5A does not substantially impact repressive Polycomb chromatin domains, but instead disrupts

87 ultra-long chromatin loops between Polycomb target genes. Our work uncovers a subset of Polycomb target genes that require distal 3D interactions for transcriptional silencing. 


\section{RESULTS}

\section{CRISPR screen of CPRC1-induced gene silencing reveals Pds5a dependence}

To identify novel regulators of PRC1/PRC2-mediated target gene silencing, we performed CRISPR-based screening. As a screening platform, we introduced stable expression of hCas9 into our TetR-CBX7 reporter line. Parental lines established a Polycomb chromatin domain with high levels of RING1B, SUZ12, H3K27me3 and H2K119ub surrounding the TetO nucleation site (Fig. 1a and Extended Data Fig. 1a). As a proof-of-principle, we infected this line with lentiviral vectors expressing either scramble sgRNA or sgRNA specific for Ring1b. We found that CRISPR mutation of Ring $1 b$ had a negligible effect on silencing induced by TetR-CBX7 (in the absence of Dox), but strongly impaired the epigenetic maintenance of silencing (in the presence of Dox, Fig. 1b), consistent with our previous observations ${ }^{31}$. Thus, our TetR-CBX7 reporter $\mathrm{mESC}$ recapitulate epigenetic Polycomb-dependent gene silencing and are sensitive to genetic perturbations.

Using this platform, we performed pooled CRISPR screens with unique molecular identifiers (UMIs), which allow analysis of mutant phenotypes at a single-cell level ${ }^{32}$. The UMI CRISPR library contained approx. 27,000 sgRNAs targeting all annotated mouse nuclear protein-coding genes with four sgRNAs per gene. Each sgRNA was paired with thousands of barcodes representing UMls, improving the signal-to-noise ratio and hit calling. hCas9-expressing TetR-CBX7 reporter mESCs were transduced with the pooled library and selected with neomycin. We used FACS to isolate GFP-positive cells (Fig. 1c and Extended Data Fig. 1b), and the unsorted population served as background control. Because GFP activation occurs at a very low frequency, we performed repeated FACS in the screen to enrich for GFP-positive cells. Relative enrichment of sgRNAs was determined by sequencing of UMIs in both populations followed by statistical analysis using MAGeCK ${ }^{33}$.

We performed a screen with reporter cells cultured without Dox (Fig. 1c), and uncovered 51 genes that were significantly enriched in the GFP-positive cell population 
118 (p-value < 0.005) (Supplementary Table 1). We also performed a separate screen of cells 119 treated with Dox for 3 days, but spontaneous GFP re-activation in some cells, 120 independently of any mutation, precluded us from identifying statistically significant hits.

121 The top hits in our screen included genes that encode subunits of cPRC1 (Cbx7,

122 Ring1b) and of PRC2 (Ezh2, Suz12, Eed), indicating that our screening approach 123 identified known genes required for TetR-CBX7-induced silencing $(C b x 7)$ and for the 124 epigenetic maintenance of silencing (Ring1b, Suz12) (Fig. 1c). Notably, Pds5a, which encodes a regulator of the cohesin complex, was the second most-significant hit in the screen. To validate this hit, we used CRISPR-Cas9 to target Pds5a independently, and observed reduced silencing in TetR-CBX7 reporter mESCs treated with Dox (Fig. 1d and Extended Data Fig. 1c). Thus, similar to Ring1b, Pds5a is required for the epigenetic maintenance of silencing induced by cPRC1.

The cohesin protein complex is composed of three core subunits, SMC1, SMC3 131 and RAD21 (also known as SCC1), which form a tripartite ring structure that entraps DNA $132{ }^{34}$. Several auxiliary cohesin proteins are critical for dynamic regulation of DNA 133 interactions. For instance, cohesin release from the DNA involves STAG1/2, WAPL and 134 PDS5A/B which associate at the interface between SMC3 and RAD21 and control ring opening ${ }^{35-43}$. In addition to Pds5a, our CRISPR screen without Dox revealed enrichment 136 of Stag2, albeit below the significance cutoff ( $p$-value $=0.045)$. Notably, a recent study 137 linked STAG2 to Polycomb domain compaction ${ }^{44}$, further supporting a potential role of 138 cohesin regulation in Polycomb-dependent gene silencing.

139 Overall, our CRISPR screen suggests that the regulation of genome topology and 140 cohesin by Pds5a promotes Polycomb-induced silencing.

\section{Loss of Pds5a results in de-repression of endogenous PRC1/PRC2 target genes}

To determine the impact of PDS5A deletion on endogenous Polycomb-dependent gene regulation, we generated Pds5a knockout mESCs using CRISPR-Cas9 (Pds5a KO).

145 In addition, we obtained a loss-of-function (LOF) mESC line harboring a disruptive gene146 trap in the second intron of the Pds5a gene $\left(P d s 5 a^{\mathrm{GT}} \mathrm{KO}\right)^{45}$. Since gene-trap disruption 
147 is reversible, we also generated a matched control mESC line in which Pds5a expression was restored (Pds5a ${ }^{\mathrm{GT}}$ WT). Pds5a knockouts as well as the rescue were confirmed by western blot (Fig. 2a). PDS5A deletion did not impact the abundance of the cohesin subunit SMC3, of the PcG proteins SUZ12 and RING1B, nor the global levels of their associated histone modifications (Fig. 2a).

Given cohesin's essential role in sister chromatid cohesion, deletion of cohesin subunits frequently impairs cell proliferation, hampering the analysis of its precise 154 function in gene regulation ${ }^{46-48}$. Although most cohesin proteins are essential for mESC viability ${ }^{46-48}$, both PDS5A and STAG2 have paralogs with redundant but not identical 156 functions that are sufficient to maintain self-renewal and proliferation ${ }^{35}$. Indeed, Pds5a KO mESC lines displayed characteristically dense colonies that could be stably maintained in culture, similar to wildtype mESCs (Extended Data Fig. 2a). Consistently, cell cycle profiles and pluripotency marker expression were highly comparable between $\mathrm{KO}$ and control mESCs, suggesting that PDS5A is largely dispensable for mESC selfrenewal and proliferation (Extended Data Fig. 2a-c).

To evaluate how endogenous Polycomb target genes are affected by PDS5A 163 deletion, we first categorized all transcription start sites (TSSs) in mESCs based on the occupancies of PRC1 and PRC2, and the enrichment of their associated histone modifications. In addition, because non-methylated CpG-islands (NMIs) have emerged 167 distinguish NMI TSSs from methylated, CpG-poor TSSs which we annotated "non-NMI" 16855 . We classified NMI TSSs with overlapping peaks of RING1B and SUZ12 as "shared 169 PRC1/PRC2 target genes", whereas NMI TSSs with RING1B peaks only were classified as "vPRC1 target genes". Notably, "shared PRC1/PRC2 target genes" displayed high

171 levels of H3K27me3 and were predominantly bound by CBX7-containing cPRC1. In 172 comparison, CBX7 was low at vPRC1 target genes which showed substantial PCGF1 173 occupancy instead. NMI TSSs lacking both RING1B (PRC1) and/or SUZ12 (PRC2) peaks 174 within $3 \mathrm{~kb}$ of their TSSs were classified as "non-PcG target genes" (Fig. 2b). 
175 Transcriptome profiling of $P d s 5 a^{\mathrm{GT}} \mathrm{KO}$ mESCs and Pds5a KO mESCs revealed 176 differential expression of $\sim 1000$ and 1500 genes, respectively (cutoff: LFC +/-0.5, padj. 177 0.05) (Fig. 2c, g, h and Extended Data Fig. 2d, g). Notably, Gene Ontology terms of 178 upregulated genes were related to developmental processes such as neurogenesis and 179 pattern specification, reminiscent of Polycomb target genes (Extended Data Fig. 2e and 180 Extended Data Fig. 2f). Although only 13\% of expressed genes in wildtype mESCs were 181 shared PRC1/PRC2 target genes, this group represented $38 \%$ and $37 \%$ of the 182 upregulated genes in Pds5a ${ }^{\mathrm{GT}} \mathrm{KO}$ and Pds5a KO mESCs, respectively (Fig. 2c). We infer 183 that silencing of this class is particularly dependent on PDS5A function. We did not 184 observe a bias in the relative distribution of gene classes among downregulated genes, 185 suggesting that PDS5A specifically promotes repression of shared PRC1/PRC2 target genes (Extended Data Fig. 2h, i).

To examine the role of PDS5A versus PRC1/PRC2 in the regulation of PRC1/PRC2 188 target gene expression, we took advantage of an mESC line that lacks the vPRC1 189 component PCGF1 and offers inducible degradation of the PRC2 component SUZ12

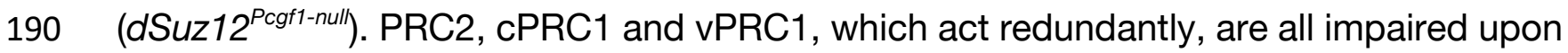
191 auxin treatment of dSuz12 Pcgf1-null mESCs. Transcriptome analysis of dSuz12 Pcgf1-null 192 mESCs treated with IAA for 48h revealed significant changes in expression of $\sim 1900$ 193 genes (cutoff: LFC +/-0.5, padj. 0.05). More than two-thirds of dysregulated genes 194 ( 1400) displayed increased expression, consistent with loss of PRC1/PRC2-mediated 195 gene silencing in mESCs. Importantly, similar to Pds5a KO mESCs, shared PRC1/PRC2 196 target genes accounted for $44 \%$ of upregulated genes. Preferential enrichment of this 197 class was specific, as the fractions of vPRC1- and non-PcG target genes remained 198 unchanged (Fig. 2d, f, g).

199 These results reveal that PDS5A plays an important role in transcriptional silencing 200 of endogenous, shared PRC1/PRC2 target genes, validating our CRISPR screening 201 results. 
PDS5A deletion has minimal effect on Polycomb repressive chromatin domains

Polycomb-mediated transcriptional silencing is linked to the formation of repressive chromatin domains marked by PRC1 and PRC2 occupancy and deposition of Polycomb-dependent histone modifications ${ }^{2,17}$. We considered that impaired silencing of endogenous PRC1/PRC2 target genes upon PDS5A deletion results from erosion of these repressive chromatin domains. To test this hypothesis, we used calibrated ChIPseq (cChIP-seq) to compare enrichment of RING1B, SUZ12, H3K27me3 and H2AK119ub between wild type and Pds5a ${ }^{\mathrm{GT}} \mathrm{KO}$ mESCs. PDS5A deletion led to a modest reduction in RING1B and H3K27me3 signals at shared PRC1/PRC2 and vPRC1 target genes, but no differences in SUZ12 and H2AK119ub enrichment across the different gene classes upon (Fig. 3 a, c, e , f and Extended Data Fig. 3a, c, f). In addition, we used ATAC-seq analysis to analyze wildtype and Pds5a ${ }^{\mathrm{GT}} \mathrm{KO}$ mESCs but did not observe significant differences in DNA accessibility, arguing that the modest difference in histone modifications does not affect the integrity of Polycomb repressive chromatin domains (Fig. 3g). Given the substantial derepression of shared PRC1/PRC2 target genes, the limited effects on repressive chromatin domains are surprising and in stark contrast with the dramatic reduction of RING1B and H2AK119ub in IAA-treated dSuz12 Pcgf1-null, which have similar silencing defects as PDS5A depletion (Fig. 3b, d and Extended Data Fig. 3b, $220 \mathrm{~d}, \mathrm{e})$.

Together, these results show that PDS5A depletion has limited impact on the

222 formation of repressive chromatin domains. The minimal reduction of PcG protein 223 binding and histone modifications appears disproportionate to the degree of aberrant 224 transcriptional activation at shared PRC1/PRC2 target genes upon loss of PDS5A, 225 suggesting that Pds5a KO impairs other mechanisms that are critical for transcriptional 226 silencing.

\section{PDS5A colocalizes with cohesin and destabilizes chromatin binding}

The PDS5A/B interaction with WAPL is critical for transient opening of the cohesin 
231 genomic distribution of PDS5A relative to the cohesin subunit RAD21, CTCF and PcG 232 proteins in wildtype mESCs. As expected, PDS5A showed extensive overlap with RAD21

233 and CTCF (Extended Data Fig. 4d-e and Extended Data Fig. 4g) ${ }^{56,57}$. In contrast, PDS5A 234 was absent from Polycomb target genes, suggesting that PDS5 indirectly promotes 235 silencing of shared PRC1/PRC2 target genes.

236 Depletion of WAPL or of both PDS5A and PDS5B, increases the cohesin residence 237 time on chromatin, resulting in continued loop extrusion, formation of larger TADs and 238 loss of compartmentalization ${ }^{37,42}$. To evaluate potential changes in the genomic 239 enrichment of cohesin between wildtype and Pds5a ${ }^{\mathrm{GT}} \mathrm{KO}$ mESCs, we performed RAD21 240 cChIP-seq (Extended Data Fig. 4d-g). Peak calling revealed three groups of RAD21 241 binding sites: "common" sites in wildtype and Pds5a GT KO mESCs ( $\mathrm{n}=15941)$, sites that 242 scored in "wildtype only" ( $n=4288)$, and sites that scored in "Pds5a GT KO only" $(n=6789)$. 243 Importantly, "common" binding sites showed increased RAD21 occupancy in Pds5aGT $244 \mathrm{KO}$ mESCs compared to wildtype, consistent with previous observations in HeLa cells 245 (Wutz et al., 2017).

246 Together, these results suggest that PDS5A acts at CTCF binding sites to release 247 cohesin from chromatin in mESCs.

PDS5A deletion increases cohesin-dependent loop extrusion, causing TAD boundary violations

251 Based on our findings above, we hypothesized that loss of PDS5A leads to a cohesin252 dependent dysregulation of 3D genome architecture that compromises the silencing of 253 endogenous, shared PRC1/PRC2 shared target genes. To investigate if PDS5A deletion 254 alters the 3D genome architecture, we performed in-situ $\mathrm{Hi}-\mathrm{C}$ on wildtype and Pds5a KO 255 mESCs. After quality control, we combined sequencing reads of Hi-C replicates 256 amounting to a total of 365 million valid unique cis-contacts per genotype 257 (Supplementary Table 2). We observed decreased interaction frequencies in Knight-Ruiz 258 (KR) ${ }^{58}$ normalized Hi-C contact matrices resulting in reduced "checkerboard" patterns 259 of alternating A and B compartments (Fig. 4a). Based on eigenvector analysis, 
compartment signal was reduced, but compartment did not switch from A to B or vice versa (Fig. 4b and Extended Data Fig. 4a, b). Reduced compartmentalization in Pds5a $\mathrm{KO}$ mESCs was further confirmed by their lower compartment strength, a related benchmark measuring interactions within compartments (A/A or $B / B$ ) compared to between compartments (A/B or B/A) (Fig. 4c). When examining relative contact probabilities (RCP) as a function of genomic distance, we found reduced compartmentalization, manifested by a decrease in very long long-range contacts ( $>5$ $\mathrm{Mb})$ in Pds5a KO mESCs relative to wildtype. Contact probabilities in the relative shortrange (50-500 kb) were also slightly reduced. In contrast, interactions in the mid- to longrange (500 kb - $5 \mathrm{Mb}$ ) were increased in Pds5a KO mESCs relative to wildtype (Fig. 4f).

Whereas PDS5A depletion reduced compartmentalization, topologically associating domain (TAD) sizes were on average larger in Pds5a KO mESCs compared to wild type mESCs (Extended Data Fig. 4c). To explore differences in TAD structure and loop formation between wildtype and $\mathrm{Pds} 5 \mathrm{a} \mathrm{KO}$, we utilized publicly available high-resolution mESC Hi-C data to identify high confidence TAD intervals and loops ${ }^{59}$. Aggregate TAD and loop analysis showed that PDS5A loss resulted in a relative contact reduction at pre-existing wildtype TADs and loops (Fig. 4d, e). We also detected increased interaction frequencies with neighboring TADs in Pds5a KO mESCs, whereas intra-TAD interactions were slightly decreased (Fig. 4g). These changes resemble those observed upon WAPL and/or PDS5A/B depletion in cancer cells, where increased cohesin residence time leads to an extension of chromatin loops, resulting in a genome-wide shift towards longer range interactions and violation of TAD boundaries ${ }^{37,42}$. Overall, our data suggest that PDS5A loss impairs cohesin unloading in mESCs.

\section{PDS5A is required to maintain a subset of Polycomb loops}

To explore how loss of PDS5A affects long-range interactions between Polycomb target genes, we used Ring1B cChIP-seq to identify 525 Polycomb (PcG) loops in wildtype mESCs (Fig. 5a). This number is similar to 336 persistent interactions identified in cohesin-depleted mESCs ${ }^{26}$, suggesting that Polycomb-associated long-range 
289 interaction account only for a small fraction of the 12425 loops detected in mESCs.

290 Further classification of Polycomb loops based on additional PcG proteins and 291 associated histone modifications revealed that virtually all of them $(476 / 525,91 \%)$ arise

292 from long-range interactions between genomic sites harboring shared PRC1/PRC2 293 target genes, consistent with recent findings ${ }^{26}$ (Fig. 5a).

Unlike non-Polycomb (non-PcG) loops, which are substantially reduced in Pds5a

295 KO mESCs, long-range interactions between shared PRC1/PRC2 target genes displayed on average only minor changes (Fig. 5a). We considered that the aberrant loop extrusion and violation of TAD boundaries that we observed in Pds5a KO mESCs could interfere with a subset of Polycomb-associated long-range interactions in a locusspecific manner. Thus, we bifurcated Polycomb loops based on interactions between anchor sites harboring upregulated (65) and not-upregulated (411 - unchanged and downregulated) shared PRC1/PRC2 target genes. Interestingly, anchor sites in wildtype mESCs that involve upregulated shared PRC1/PRC2 target genes are engaged in stronger loops when compared to the anchor sites that do not involve upregulated genes (Fig. 5b). Strikingly, upon PDS5A deletion, the interaction frequency at upregulated anchor sites was dramatically reduced, whereas interactions between not-upregulated anchor sites were relatively unaffected, similar to the class average (Fig. 5b). These

results suggest that local dysregulation of cohesin-mediated chromosome architecture interferes with long-range interactions at a subset of shared PRC1/PRC2 target genes, which in turn compromises gene silencing.

Repressive polycomb loops crossing ultra-long distances are sensitive to cohesin dysfunction

To understand what defines the subset of Polycomb loops that are vulnerable to cohesin dysfunction, we first compared chromatin modifications between anchor sites of upregulated and not-upregulated PRC1/PRC2 target genes. We noticed that at

316 Polycomb loops of upregulated PRC1/PRC2 target genes, only one of the two anchor

317 sites was associated with loss of gene silencing. To investigate potential differences in 
318 the repressive chromatin modifications, we separated the two anchor sites into not319 upregulated (left) and upregulated (right) and compared PcG protein occupancy and 320 associated histone modifications (Fig. 5c and Extended Data Fig. 5a). Anchor sites of 321 not-upregulated shared PRC1/PRC2 target genes served as the control dataset. 322 Surprisingly, despite differential expression we found that upregulated (right) and notupregulated anchor sites (left) had comparable repressive chromatin domains with 324 similar reduction in Ring1B occupancy and H3K27me3 upon PDS5A deletion (Fig. 5c 325 and Extended Data Fig. 5a). Chromatin modifications at upregulated anchor sites were 326 also similar to those at anchor sites of not-upregulated shared PRC1/PRC2 target genes. 327 Together, these results corroborate our genome-wide analysis revealing minimal 328 reduction of repressive chromatin modifications at shared PRC1/PRC2 target genes and 329 strongly suggest that reduced long-range interactions and loss of gene silencing in Pds5a KO mESCs are largely uncoupled from changes in Polycomb chromatin domains.

Next, we explored if sensitivity to cohesin deregulation is linked to the distance between Polycomb anchor sites. Comparison of loop sizes of upregulated and notupregulated shared PRC1/PRC2 target genes revealed a striking difference: loops between anchor sites of upregulated shared PRC1/PRC2 target genes were substantially longer than loops between anchor sites of not-upregulated or all shared PRC1/PRC2 336 target genes (Fig. 5d). Not surprisingly, this length bias of loops was also reflected in a 337 greater number of TADs within A and B compartments traversed by anchor sites of 338 upregulated shared PRC1/PRC2 target genes. Based on these results we conclude that 339 ultra-long Polycomb loops are most vulnerable to cohesin dysfunction. We speculate 340 that traversing a greater number of TADs increases the probability of interference by 341 cohesin-mediated loop extrusion and TAD boundary violations. Importantly, by 342 uncoupling loss of Polycomb loops from changes in repressive chromatin modifications, 343 these results reveal a subset of shared PRC1/PRC2 target genes that depend on long344 range interactions in silencing. 


\section{Loss of repressive Polycomb loops is linked to cohesin-mediated insulation gain}

To uncover the potential mechanism by which increased cohesin residence could interfere with repressive ultra-long loops between shared PRC1/PRC2 target genes, we defined regions in the genome with significant local changes in 3D chromosome architecture. Specifically, we calculated insulation scores ${ }^{60}$ in $250 \mathrm{~kb}$ bins across the genomes of wildtype and Pds5a KO mESCs. Insulation scores in most bins ( 41000) were unchanged upon PDS5A deletion (Fig. 6a). Additionally, we identified $\sim 17000$ bins with significant reduction in insulation in Pds5a KO mESCs, suggesting loss of TAD boundaries in response to increased cohesin residence time (Extended Data Fig. 6a).

Intriguingly, we identified $\sim 1400$ genomic regions that gained insulation in $P d s 5 a$ KO mESCs (Fig. 6a). We reasoned that these newly formed insulation sites could interfere with repressive ultra-long loops between shared PRC1/PRC2 target genes. To explore this scenario, we analyzed the distances between newly formed insulation sites and Polycomb target genes. Strikingly, new insulation sites are located significantly closer to upregulated shared PRC1/PRC2 target genes than to all other classes of Polycomb and non-Polycomb genes (Fig. 6b). These data suggest that upregulation of a subset of shared PRC1/PRC2 target genes results from proximal changes in cohesinmediated 3D chromosome architecture that cause a loss of ultra-long Polycomb loops.

One prominent example of such cohesin-dependent dysregulation is the insulation gain region located between $D / x 2$ and the HoxD gene cluster (Fig. 6c). In wildtype mESCs, Dlx2 forms strong interactions traversing approximately $3 \mathrm{Mb}$ with the HoxD gene cluster. Upon PDS5A deletion, these long-range interactions are lost and DIx2 expression is upregulated by more than 8-fold (Fig. 6c and Fig. 2e), yet PcG protein occupancy and associated histone modifications are either unaffected or only marginally reduced at DIx2 and the HoxD gene cluster (Fig. 5c and Fig. 3c, d). Instead, PDS5A deletion leads to a gain in insulation with increased cohesin binding near the Sp9 gene, which is located between D/x2 and the HoxD gene cluster. Virtual 4-C viewpoints from the insulation gaining region (v2), as well as from $D / x 2$ (v1) and the HoxD gene cluster (v3) reveal increased longer-range contacts in Pds5a KO mESCs, consistent with 
374 aberrant extension of chromosomal loops (Fig. 6c). This shift towards longer-range 375 interactions is captured in $\mathrm{Hi}-\mathrm{C}$ matrices as strengthening of two domains in between 376 DIx2 and the HoxD gene cluster (dashed triangles) (Fig. 6c). We speculate that aberrant 377 extension of chromosomal loops and strengthening of ectopic interaction domains 378 comes at the expense of the ultra-long Polycomb loop between DIx2 and the HoxD gene 379 cluster. A similar example showcasing how insulation gain might interfere with Polycomb 380 long-range interaction and gene repression is represented by contacts between Hoxb13 and Ppp1r1b (Extended Data Fig. 6b).

382 Taken together our results argue that Polycomb loops are critical for silencing a 383 subset of shared PRC1/PRC2 target genes. Pds5a KO causes changes in cohesin384 dependent 3D genome architecture that perturb competing loops mediated by cPRC1. 385 Notably, our data demonstrate that the resulting loss of Polycomb loops is linked to loss 386 of silencing despite the presence of large repressive Polycomb chromatin domains. 387 Thus, Polycomb repression takes place in a delicate spatial equilibrium with cohesin388 dependent nuclear architecture, that is essential to maintain robust silencing at shared PRC1/PRC2 target genes.

\section{DISCUSSION}

Here, we used a CRISPR-mutagenesis screen to identify novel regulators of cPRC1-induced gene silencing which revealed the cohesin regulatory subunit PDS5A. Subsequent KO in mESCs confirmed that PDS5A is required for repression of a subset of canonical Polycomb target genes. Notably, loss of developmental gene silencing is mostly uncoupled from changes in Polycomb repressive chromatin domains. Instead, PDS5A loss affects cohesin-dependent genome architecture which in turn perturbs competing loops mediated by cPRC1. Hence, our results strongly argue that ultra-long

399 Polycomb loops are critical for robust silencing at a subset of canonical Polycomb target 400 genes.

401 Intriguingly, even though the screen was performed in the absence of Dox, some 402 of the genes that were identified (Ring1b, Suz12, Pds5a) are required for reporter gene 
403 silencing in the Polycomb in vivo assay only following Dox-dependent release of TetR404 CBX7. We infer that the screen, which involved repeated FACS, relied on genes involved 405 in the initiation or maintenance of reporter silencing. Overall, we predict that the 406 epigenetic inheritance of reporter silencing involves a repressive Polycomb chromatin 407 domain that engages in ultra-long interactions with shared PRC1/2 target genes. By 408 causing cohesin dysfunction, PDS5A loss would disrupt the spatial integration of the silenced reporter locus from the existing network of Polycomb loops.

Previous reports have linked gene mutations of cohesin subunits to defects in 411 Polycomb-dependent gene silencing, but the mechanisms had remained unclear. For 412 example, a genetic screen for dominant suppressors of Polycomb-dependent silencing 413 in Drosophila revealed several mutants in the wapl gene ${ }^{61}$. In mammals, WAPL is a 414 binding partner of PDS5A/B and the major regulator of cohesin off-loading and positioning ${ }^{37,42,56}$. Pds5a KO mice exhibit developmental abnormalities, including skeletal malformations ${ }^{62}$, that resemble the patterning defects of Polycomb mutant mice ${ }^{24,63,64}$.

The capacity of $\mathrm{CPRC} 1$ to form 3D chromatin interactions that contribute to gene silencing has been previously demonstrated in Drosophila ${ }^{21,28}$. However, contributions 419 to gene silencing by CPRC1 in mammalian systems has been controversial ${ }^{18,19,29,30,65,66}$. 420 The repertoire of divergent PRC1 and PRC2 complexes increases dramatically from fly 421 to mammals. Emerging hierarchies of Polycomb signaling cascades put vPRC1 and 422 H2AK119ub front and center of Polycomb repression (reviewed in ${ }^{17}$ ). Meanwhile, the 423 repressive capacities of canonical pathways via PRC2, H3K27me3 and cPRC1 have 424 been questioned, as Pcgf2/4-containing cPRC1 complexes have been shown to be 425 largely dispensable in mESCs ${ }^{18,19,67}$. Nevertheless, the repressive capabilities of cPRC1 $^{2}$ have been clearly demonstrated $29,31,66,68,69$.

427 Multiple studies in mammals have confirmed that $\mathrm{CPRC} 1$ mediates formation of 428 chromatin loops underlying a 3D nuclear network of Polycomb target genes ${ }^{12,22,27,70,71}$. 429 Although the exact mechanisms that mediate these interactions remain unknown, PHC 430 proteins, exclusively found in $\mathrm{CPRC} 1$, have been proposed to mediate these distal 431 interactions via oligomerization of their SAM-domains ${ }^{24,25}$. Importantly, recent studies 
432 showed that CPRC1-mediated distal interactions and compaction of Polycomb domains

433 are interdependent with genome organization by cohesin ${ }^{26,4426,44}$. STAG2-containing

434 cohesin complexes have been shown to contribute to compaction of Polycomb 435 chromatin domains ${ }^{44}$ and in Ewing sarcoma cells STAG2 depletion leads to a reduction 436 in H3K27me3 and deregulation of PRC2 target genes ${ }^{72}$. Furthermore, acute depletion of 437 cohesin in mESCs resulted in stabilization of Polycomb chromatin loops and 438 enhancement of transcriptional repression. These reports support a model that cohesin439 mediated chromosome structure in the interphase nucleus normally restricts the 440 formation of Polycomb looping ${ }^{26}$. Here, we discovered that ultra-long Polycomb loops 441 are preferentially affected by cohesin dysfunction caused by Pds5a KO. This finding is 442 consistent with overarching principles in nuclear organization: compartmental domains 443 form between similar chromatin states, leading to the checkerboard pattern observed in $444 \mathrm{Hi}-\mathrm{C}$ data, where $\mathrm{A}$ and $\mathrm{B}$ compartments tend to interact with other $\mathrm{A}$ and $\mathrm{B}$ 445 compartments rather than A with B or vice versa. Cohesin- and CTCF-dependent loop 446 formation on the one hand facilitates interaction frequencies within identical 447 compartmental domains, but on the other hand loops across TADs of $A$ and/or B 448 compartments restrict their segregation. Thus, CTCF depletion removes this restriction 449 of segregation, leading to increased compartmentalization despite a loss of loops ${ }^{42,73-76 .}$. 450 In addition, stabilizing cohesin enhances loop extrusion and increases the restriction of 451 compartment segregation ${ }^{37,42,75}$. Hence, this model could explain why PDS5A loss 452 preferentially affects longer loops, that might be formed by such natural interaction of 453 similar chromatin domains over very long distances that usually are not disturbed by 454 cohesin loop extrusion ${ }^{75}$.

$455 \quad$ Nevertheless, to our knowledge a causal link between Polycomb-mediated long456 range interactions and repression of Polycomb target genes has not been described. 457 Polycomb target genes are generally located within the active $(A)$ compartment in the 458 nucleus of mESCs and thereby in spatial proximity to actively transcribed genomic 459 regions ${ }^{77}$. We speculate that Polycomb-dependent silencing of canonical target genes 460 involves multiple parallel mechanisms including repressive histone modifications and 
461 long-range interactions. By uncoupling loss of Polycomb silencing and loop interactions

462 from changes in repressive chromatin modifications, our data argue that 3D organization

463 by itself has repressive function potentially by tethering PRC1/PRC2 target genes away

464 from transcriptional co-activators and/or RNA Polymerase. Therefore, when combined

465 with repressive chromatin modifications, which promote Polycomb feedback 466 mechanisms, spatial aggregation by Polycomb loops would effectively enhance robust 467 gene silencing.

468 The relative contribution of each of these mechanisms is likely locus-specific and 469 may vary in different cell types. For example, alternative incorporation of paralogous 470 cPRC1 subunits, such as CBX proteins or PHC proteins may influence the specific 471 regulation of Polycomb 3D network formation as a mechanism of repression. Hence, 472 future studies are needed to discern how cPRC1 and Polycomb-dependent genome 473 architecture control target gene silencing in the context of different cell types and in 474 disease.

475

\section{Acknowledgements:}

477 We are grateful to all members of the Bell, Farnham and Jadhav laboratories, as well as 478 Alexander Stark, Martin Leeb, Jan Michael Peters, Gordana Wutz, Diana Hargreaves, 479 Jesse Dixon, Suhn Rhie and Geoffrey Fudenberg for feedback and discussions. We 480 especially thank llya Flyamer for sharing $\mathrm{Hi}-\mathrm{C}$ protocols and advice on Hi-C data 481 analysis. We thank Jan Michael Peters and Gordana Wutz for sharing antibodies. We 482 thank the Vienna Biocenter Core Facility Next Generation Sequencing. The 483 GMI/IMBA/IMP Scientific Service units and the BioOptics facility. We thank Life Science 484 Editors for editorial assistance.

486 Funding:

487 O.B. and U.E. were supported by the Austrian Academy of Sciences. O.B. was 488 supported by the New Frontiers Group of the Austrian Academy of Sciences (NFG-05), 489 the Human Frontiers Science Programme Career Development Award (CDA00036/2014- 
C), and start-up funding from the Norris Comprehensive Cancer Center at Keck School

491 of Medicine of USC.

492

493 Author contributions:

494 D.B., H.F.M., O.B. initiated and designed the study. D.B., H.F.M. generated cell lines.

495 R.Y. generated parental cell lines. U.E., G.M. provided the CRISPR sgRNA library and

496 helped with the screen design. D.B., H.F.M. performed CRISPR-Cas9 genetic screen.

497 J.W. and G.M. analyzed CRISPR-Cas9 genetic screen data. D.B. performed molecular 498 biology, RNA-seq, ChIP-seq and Hi-C experiments. S.G., Q.Z. participated in 499 experiments. RNA-seq, ChIP-seq and Hi-C data analysis was conducted by D.B. O.B. 500 supervised all aspects of the project. The manuscript was prepared by D.B. and O.B..

501 All authors discussed results and commented on the manuscript.

502

503

Data, Material and Code availability:

504 All NGS data reported in this study has been deposited at the Gene Expression Omnibus

505

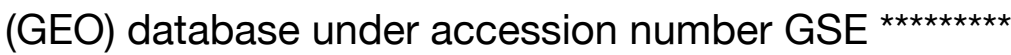

506

507

Competing interests:

508 The authors declare that they have no competing interests.

509

510

\section{METHODS}

\section{Cell lines}

513 All cell lines used directly in this study or for generating mutants were diploid mESCs 514 derived from originally haploid HMSc2 termed AN3-12 ${ }^{45}$. TetR-CBX7 reporter mESCs 515 with 7x TetO DNA binding sites flanked by GFP and BFP reporter genes were previously 516 described ${ }^{31}$. Pds5a gene-trap (GT) KO and its corresponding wild type mESCs with 517 genetrap insertion in the non-disruptive orientation were aquired from the Haplobank 518 repository (Cell IDs: $10388 \mathrm{IH}$ and $10388 \mathrm{MH})^{45}$. 


\section{Cell culture conditions}

521 All mESCs were cultivated without feeders in high-glucose-DMEM (Corning 10-013-CV)

522 supplemented with 13.5\% fetal bovine serum (Corning 35-015-CV), $10 \mathrm{mM} \mathrm{HEPES} \mathrm{pH}$

5237.4 (Corning, 25-060-Cl), 2 mM GlutaMAX (Gibco, 35050-061), 1 mM Sodium Pyruvate

524 (Corning 25-000-Cl), 1\% Penicillin/Streptomycin (Sigma, P0781), 1X non-essential

525 amino acids (Gibco, 11140-050), 50 mM $\beta$-mercaptoethanol (Gibco, 21985-023) and

526 recombinant LIF. Cells were incubated at $37^{\circ} \mathrm{C}$ and $5 \% \mathrm{CO}_{2}$ and were passaged every

52748 hours by trypsinization in 0.25\% 1x Trypsin-EDTA (Gibco, 25200-056). In order to

528 reverse of TetR-CBX7 fusion protein binding $1 \mu \mathrm{g} / \mathrm{ml}$ Doxycycline (Sigma, D9891) was

529 added to cell culture medium.

530

531 Generation of CRISPR-Cas9 mutants

532 TetR-CBX7 RING1B and PDS5a loss of function (LOF) as well as An3-12 Pds5a LOF

533 mutants were generated using CRISPR-Cas9 technology. sgRNAs targeting Pds5a (5'-

534 TGTCTCTGCAGAGTGGAACG- 3') or Ring1b (5'- ACAAAGAGTGTCCTACCTGT -3')

535 were introduced into a modified version of the vector plasmid pX330-U6-Chimeric_BB-

536 CBh-hSpCas9 (Addgene \#42230) that yields a BFP marker for selection (Gift by J.

537 Zuber). Plasmid transfection was achieved by electroporation with the NEON

538 transfection system (Invitrogen, MPK5000). 36 hours post transfection cells were FACS

539 sorted for Cas9-BFP and 1000-2000 cells seeded for clonal expansion on a 15cm plate.

540 7-10 days later colony forming clones were individually picked, the targeted loci

541 genotyped and loss of function confirmed by western blot.

542

\section{Hoechst-staining}

544 For cell cycle profiling mESCs were trypsinized and genomic DNA was stained with

545 Hoechst 33342 (20 mM; Thermo Fisher Scientific Cat. \# 62249) for $30 \mathrm{~min}$ at $37^{\circ} \mathrm{C}$ and

$5465 \% \mathrm{CO}_{2}$. Hoechst immunofluorescence was measured by flow cytometry on a FACSAria

547 II cell sorter (BD Biosciences). 
548

549

550

551

552

553

554

555

556

\section{7}

558

559

560

561

562

563

564

565

\section{6}

567

568

569

570

571

572

573

574

575

576

\section{AP-staining}

One thousand cells were seeded and grown to form colonies at low density on $15 \mathrm{~cm}$ tissue culture dishes for 7 days. On day 7 , dishes were washed with $100 \mathrm{mM}$ tris $(\mathrm{pH} 8)$ and AP activity assay was performed using the VECTOR Blue AP Substrate Kit (Vector Laboratories, VECSK-5300) according to the manufacturer's instructions. Following AP staining, stained colonies were fixed in $4 \%$ formaldehyde overnight. Plates were rinsed with $1 \times$ PBS the following day and images taken on a brightfield microscope (EVOS XL Core system).

\section{Pluripotency marker staining}

To assess pluripotency of PDS5a loss of function mutants, we applied intracellular staining of OCT3/4, SOX2 and SSEA1. Single cell suspensions of wild type and Pds5a KO mESCs were permeabilized and fixed using the fixation/permabilization buffer (R\&D systems), washed twice with $1 \times$ PBS and stained using the H/MM pluripotent Stem Cell Multi-Color Flow Cytometry kit (R\&D Systems) according to vendor's protocol. Flow cytometry data was collected on an Attune NxT equipped with Attune NxT v3.1 acquisition software. Final data analysis was performed using FlowJo (10.7.1).

\section{Western blot}

10 million mESCs were subsequently lysed in Buffer $\mathrm{A}(25 \mathrm{mM}$ Hepes pH 7.6, $5 \mathrm{mM}$ $\mathrm{MgCl}_{2}, 25 \mathrm{mM} \mathrm{KCl}, 0.05$ mM EDTA, 10\% Glycerol, 1 mM DTT, 1 mM PMSF, $1 \times$ Complete Mini protease inhibitor, Roche) resuspended in RIPA buffer (150 mM NaCl, $1 \%$ triton, $0.5 \%$ sodium deoxy-cholate, $0.1 \%$ SDS, $50 \mathrm{mM}$ Tris $\mathrm{pH}$ 8.0). Lysates were homogenized by sonication using a Bioruptor Pico (Diagenode) and concentration determined by Bradford assay (Biorad). 4x non-reducing Laemmli SDS sample buffer (Alfas Aesar, \#J63615AD), $10 \mathrm{mM}$ final DTT and $0.5 \%$ final BME were added to $20 \mu \mathrm{g}$ total protein/sample and boiled at $95^{\circ} \mathrm{C}$ for $5 \mathrm{~min}$. Samples were separated on NuPAGE 4$12 \%$ Bis-Tris gels (Invitrogen) in Bis-Tris running buffer (Novues Biologicals) and transferred on a Merck Chemicals Immobilon-FL Membrane (PVDF $0.45 \mu \mathrm{m}$ ). After 
577 blocking the membranes (5\% non-fat dry milk in $1 \times$ PBS, $0.1 \%$ Tween 20) the blots were 578 incubated $\mathrm{o} / \mathrm{n}$ with the primary antibodies in 5\% non-fat dry milk in $1 \times$ PBS and $0.1 \%$

579 Tween 20. Antibodies used: PDS5a (Millipore Sigma \#SAB2101764) 1:1000; RING1B

580 (Cell Signaling D22F2) 1:1000; SMC3 (Bethyl Laboratories A300-060A) 1:2000; SUZ12

581 (Cell Signaling D39F6) 1:1000; LAMIN B1 (Abcam ab16048) 1:15000; H2AK119ub (Cell

582 Signaling D27C4) 1:20000; H3K27me3 (Diagenode p069-050) 1:1000. Next, the

583 membranes was incubated with corresponding secondary IRDye 800CW Goat anti-

584 Rabbit IgG (H+L) (LICOR) or IRDye 680RD Goat anti-Mouse IgG (H+L) (LICOR) antibodies

585 and imaged on an Odyssey CLx Near-Infrared Imaging System (LICOR).

586

587 Genetic CRISPR-Cas9 screen

588 For the genetic CRISPR-Cas9 mutagenesis screen, EF1a promoter driven hSpCas9 with

589 a hygromycin resistance marker (modified version of Addgene \#52961) was stably 590 integrated via lentiviral transduction into the previously described TetR-CBX7 reporter 591 mESC cell line, which contains 7x TetO DNA binding sites flanked by GFP and BFP 592 reporter genes ${ }^{31}$.

593 For CRISPR-Cas9 mutagenesis, a sgRNA library targeting 6,560 nuclear factors with 4 594 sgRNAs per gene and 112 nontargeting controls was utilized (described in ${ }^{32}$. For 595 retroviral library generation, the barcoded plasmid library of sgRNAs, containing 596 neomycin resistance for selection, was packaged in PlatinumE cells (Cell Biolabs) 597 according to the manufacturer's recommendations.

598300 million TetR-CBX7 reporter mESCs were infected with a 1:10 dilution of the 599 harvested virus-containing supernatant PlatinumE cell medium for 24 hrs in the presence 600 of $2 \mu \mathrm{g} / \mathrm{ml}$ polybrene (Santa Cruz Biotechnology, SACSC-134220). The 300 million cells 601 were divided into 3x 100 million sets (10x $15 \mathrm{~cm}$ plates of 10 million cells each) that were 602 treated as 3 separate replicates throughout entirety of the mutagenesis screen and 603 sgRNA NGS sequencing. 24 hrs post infection, neomycin-resistance selection was 604 started on the infected cells by addition of G418 (Gibco) at $0.5 \mathrm{mg} / \mathrm{ml}$. After $24 \mathrm{hrs}$ of 605 selection, each replicate was expanded from $1015-\mathrm{cm}$ dishes to 20 dishes. 
606 Subsequently, for the duration of the neomycin selection cells were always maintained 607 at a minimum of 300 million cells. After 5 days and completion of G418 selection, half of 608 the cells were cultured in mESC medium containing doxycycline for 3 days and the other 609 half without doxycycline. GFP positive cell populations of both doxycycline treated and 610 untreated populations were sorted on a FACSAria II cell sorter (BD Biosciences) and flow 611 cytometry data analyzed with FlowJo software. Unsorted mutant populations were

612 served as background controls. Genomic DNA was isolated from GFP-positive sorted 613 and unsorted cells, their sgRNA cassettes amplified by PCR and subjected to NGS sequencing on an Illumina HiSeq 2500. Data analysis was performed as previously described in (Michlits 2017) and gene enrichment determined using MAGeCK ${ }^{33}$.

\section{RNA-seq}

6185 million mESCs were trypsinized and collected by centrifugation. Resulting cell pellets 619 were washed in 1x PBS and resuspended in 1x DNA/RNA protection reagent (Monarch 620 Total RNA Miniprep Kit, NEB). Subsequently, cells were lysed and total RNA extracted 621 following the mammalian cell protocol including optional on-column DNase I treatment. 622 For RNA-seq library preparation, $1 \mu \mathrm{g}$ of total RNA per sample was enriched for poly-A 623 using the NEBNext Poly(A) mRNA Magnetic Isolation Module (NEB, E7490) and final 624 RNA-seq libraries generated using the NEBNext Ultra II Directional RNA Library Prep kit 625 (NEB, E7760 and NEBNext Multiplex Oligos (NEB, E7335/E7500). Final libraries were 626 sequenced as $150 \mathrm{bp}$ paired-end reads on the Illumina HiSeq platform.

627

\section{RNA-seq Data Analysis}

629 Raw paired-end RNA-seq reads were aligned to the mm10 genome using STAR-2.6.1c

$630{ }^{78}$. Overlap of STAR-aligned reads with genes was performed using HTSeq count 631 function ${ }^{79}$ with stranded=reverse option and the GRCm38 version 94 GTF file. The 632 HTseq count matrix was pre-filtered to exclude genes with a read count below 10. 633 Differential gene expression analysis was performed using DESeq2 ${ }^{80}$ using the "apeglm" 634 method ${ }^{81}$ for LFC shrinkage. We applied a threshold of p-adj $<0.05$ and fold change $>$ 
6350.5 or -0.5 for gene expression changes to be considered significant. Visualization of 636 RNA-seq data was performed using custom R scripts and ggplot2. Gene ontology 637 analysis for significantly deregulated genes was performed using custom R scripts and 638 clusterProfiler reference ${ }^{82,83}$.

639

\section{Calibrated ChIP-seq (cChIP) and ChIPCap-seq}

64130 million mESCs and HEK293T cells were collected, washed once in 1x PBS and 642 crosslinked for 7 min in 1\% formaldehyde. The crosslinking was quenched by addition

643 of $125 \mathrm{mM}$ glycine and incubated on ice. The crosslinked cells were pelleted by centrifugation for $5 \mathrm{~min}$ at $1200 \mathrm{~g}$ at $4{ }^{\circ} \mathrm{C}$. Nuclei were prepared by washes with NP-Rinse buffer 1 (10 mM Tris pH 8.0, 10 mM EDTA pH 8.0, 0.5 mM EGTA, 0.25\% Triton X-100) followed by NP-Rinse buffer 2 (10 mM Tris pH 8.0, 1 mM EDTA, 0.5 mM EGTA, 200 mM $\mathrm{NaCl}$ ). Afterwards, the nuclei were washed twice with shearing buffer (1 mM EDTA pH 8.0, $10 \mathrm{mM}$ Tris- $\mathrm{HCl} \mathrm{pH} 8.0,0.1 \%$ SDS) and subsequently resuspended in $900 \mu \mathrm{L}$ shearing buffer with added $1 \times$ protease inhibitors complete mini (Roche). Chromatin was sheared by sonication in $15 \mathrm{ml}$ Bioruptor tubes (Diagenode, C01020031) with $437.5 \mathrm{mg}$ sonication beads (Diagenode, C03070001) for 6 cycles (1 $\mathrm{min}$ on/1 $\mathrm{min}$ off) on a Bioruptor Pico sonicator (Diagenode). For each ChIP reaction 4 \% HEK293T-derived human spike-in lysate was combined with mESC lysate and incubated in 1x IP buffer (50 mM HEPES/KOH pH 7.5, 300 mM NaCl, 1 mM EDTA, 1\% Triton X-100, 0.1\% DOC, $0.1 \%$ SDS), with following appropriate antibodies at $4{ }^{\circ} \mathrm{C} \mathrm{o} / \mathrm{n}$ a rotating wheel: H3K27me3 (Diagenode, C15410195), RING1B (Cell Signaling, D22F2), PDS5a (Millipore

657 Sigma \#SAB2101764), SUZ12 (Cell Signaling D39F6), H2AK119ub (Cell Signaling 658 D27C4), PCGF1 (Abcam ab202395), RAD21 (Abcam ab992), CTCF (Millipore 070729).

659 Antibody-bound chromatin was captured using Dynabeads protein G beads 660 (Thermofisher \#10004D) for 4 hours at $4^{\circ} \mathrm{C}$. ChIP washes were performed as described 661 previously ${ }^{51}$. ChIPs were washed 5x with 1x IP buffer ( 50 mM HEPES/KOH pH 7.5, 300 $662 \mathrm{mM} \mathrm{NaCl}$, I mM EDTA, 1\% Triton-X100, 0.1\% DOC, 0.1\% SDS), or 1.5x IP buffer for 663 H3K27me3 and H2AK119ub, followed by 3x washes with DOC buffer (10 mM Tris pH 8, 
$6640.25 \mathrm{mM}$ LiCl, $1 \mathrm{mM}$ EDTA, 0.5\% NP40, 0.5\% DOC) and 1x with TE/50 mM NaCl. ChIP 665 DNA was eluted $2 x$ in elution buffer (1\% SDS, $0.1 \mathrm{M} \mathrm{NaHCO}_{3}$ ) at $65^{\circ} \mathrm{C}$ for 20 min, RNase 666 A treated for 30 min at $37^{\circ} \mathrm{C}$, Proteinase $\mathrm{K}$ treated for $3 \mathrm{hrs}$ at $55^{\circ} \mathrm{C}$ and crosslinks were reversed o/n at $65^{\circ} \mathrm{C}$. The following day, ChIP samples and corresponding inputs were 668 purified by $\mathrm{PCl}$ extraction and DNA precipitation.

cChIP-seq and ChIPCap-seq library preparation

671 Libraries were prepared using the NEXTflex ChIP-Seq kit (Bio Scientific) following the

672 "No size-selection cleanup" protocol. Libraries were purified using Agencourt AMPure 673 XP (Beckman Coulter) and amplified using the KAPA Real-Time Library Amplification Kit 674 (KAPABiosystems) following the manufacturer's instructions.

675 ChIPCap-seq libraries were prepared identically to ChIP-seq libraries. After PCR 676 amplification the libraries were enriched for loci of interest using the MYbaits kit DNA 677 capture target enrichment system (Arbor Biosciences) according to manufacturer's 678 manual. 120 nucleotides long MYbaits sequence capture probes (Arbor Biosciences)

679 were custom designed against 25 mm9 genomic loci (Supplementary Table 3).

680 Library quality control including determination of average size and concentration was 681 performed prior to sequencing by commercial Next Generation Sequencing providers. 682 NGS libaries were eventually sequenced as $150 \mathrm{bp}$ paired-end reads on the Illumina 683 HiSeq platform.

684

685

\section{ChIP-seq Data Analysis}

686 Raw reads were mapped to the custom concatenated mouse (mm10) and spike-in 687 human (hg38) genome sequences using bowtie 2 with "-no-mixed" and "no-discordant" 688 options (Langmead and Salzberg, 2012). Subsequently, low quality reads were filtered 689 using SAMtools ${ }^{84}$, duplicated reads were discarded with the Picard toolkit 690 (http://broadinstitute.github.io/picard/) and only unique mapped reads were retained.

691 For visualization uniquely mapped mouse reads were normalized by random 692 subsampling with samtools using calibration factors calculated from the corresponding 
693

694

695

696

697

698

699

700

701

702

703

704

705

706

707

708

709

710

711

712

713

714

715

716

717

718

719

720

721

hg38 spike-in reads as described previously ${ }^{19,85,86}$. High correlation between replicates was confirmed using multiBamSummary and plotCorrelation functions from deepTools ${ }^{87}$ before merging for visualization and downstream analysis. Genome coverage tracks (bigWig files) were produced with MACS2' pileup function ${ }^{88}$ and heatmaps and profile plots generated with deepTools ${ }^{87}$.

Peaks were called on each replicate independently using MACS2 ${ }^{88}$. Peaks overlapping with a custom-build blacklist were discarded to remove sequencing artifacts and only peaks called in both replicates were retained for downstream analysis.

\section{In Situ Hi-C}

Hi-C was performed as previously described ${ }^{77}$ with modifications described in ${ }^{22}$ as following: 5 million mESCs were crosslinked in 1\% formaldehyde for 10 mins before the reaction was quenched by adding $0.2 \mathrm{M}$ final glycine. Cells were permeabilized in lysis buffer (0.2\% IGEPAL, $10 \mathrm{mM}$ Tris- $\mathrm{HCl}$ pH 8.0, $10 \mathrm{mM} \mathrm{NaCl}, 1 \times$ Halt Protease inhibitor cocktail) and nuclei isolated in NEBuffer 3 supplemented by $0.3 \%$ SDS at $62{ }^{\circ} \mathrm{C}$ for 10 min. SDS was quenched with $1 \%$ Triton $\mathrm{X}-100$ at $37^{\circ} \mathrm{C}$ for $60 \mathrm{~min}$, the nuclei pelleted and resuspended in $250 \mu \mathrm{l}$ of 1x Dpnll buffer with $600 \mathrm{U}$ Dpnll (NEB). Following o/n digestion at $37^{\circ} \mathrm{C}, 200 \mathrm{U}$ were added for $2 \mathrm{hrs}$. Dpnll was inactivated for $20 \mathrm{~min}$ at $65^{\circ} \mathrm{C}$ before the DNA ends were filled-in and biotin-marked using Klenow, d(C/G/T)TPs and biotin-14-dATP for $90 \mathrm{~min}$ at $37^{\circ} \mathrm{C}$. Proximity ligation was performed using T4 DNA ligase (NEB) for $4 \mathrm{hrs}$ at room temperature. Subsequently, nuclei were spun down, resuspended in $200 \mu \mathrm{mQ}$ water and digested with proteinase $\mathrm{K}$ for $30 \mathrm{~min}$ at $55^{\circ} \mathrm{C}$ in presence of $1 \%$ SDS. For crosslink reversal $1.85 \mathrm{M}$ final $\mathrm{NaCl}$ was added and samples incubated at $65^{\circ} \mathrm{C} \mathrm{o} / \mathrm{n}$. The next day, sample DNA was ethanol precipitated and sheared in $500 \mu \mathrm{L}$ sonication buffer (50 mM Tris pH 8.0, 0.1\% SDS, $10 \mathrm{mM}$ EDTA) on a Bioruptor Pico sonicator (Diagenode). DNA was then concentrated on Amicon ultra $0.530 \mathrm{~K}$ filter units (Millipore), biotin-pulldown performed using MyOne Streptavidin T1 beads (Life technologies, 65602) and used for NGS library preparation. DNA ends were repaired, biotin removed from unligated ends and NEXTFLEX DNA barcoded adapters (Perkin 
722 Elmer) were ligated. Desired PCR cycle numbers were determined in test endpoint PCRs

723 using Q5 DNA polymerase (NEB M0491L). Final HiC libraries were generated from 4-6

724 individual PCR reactions, which were pooled and subjected to cleanup and size725 selection using AMPure beads (Beckman Coulter A63882). Samples were first test 726 sequenced to check library quality before selected $\mathrm{Hi}-\mathrm{C}$ ibraries were sequenced at 727 greater depth (Supplementary Table 2).

728

729 Hi-C data analysis

$730 \mathrm{Hi}-\mathrm{C}$ data were analyzed using the HiC-Pro (2.11.1) pipeline ${ }^{89}$. Read mapping to the $731 \mathrm{~mm} 10$ genome was performed using bowtie $2^{90}$ within the HiC-Pro pipeline. PCR and 732 optical duplicates as well as reads with $\mathrm{MAPQ}<30$ were removed. Filtered valid $\mathrm{HiC}$ 733 contact data was binned and raw and ICE normalized hic contact matrices were 734 generated. We also produced balanced single and multi-resolution .cool and .mcool 735 cooler files for visualization in HiGlass. Virtual 4C tracks were obtained using the 736 hicPlotViewpoint function of HiCExplorer ${ }^{91-93}$. For reference the same analysis was 737 applied to published deep Hi-C data from mESCs ${ }^{59}$. To obtain wild type mESC TAD and 738 loop information we applied juicer tools "94 "arrowhead" and "HiCCUPS" to the Bonev 7392017 mESC dataset. To delineate A and B compartment information, "Eigenvector" 740 function of juicer tools was applied to 250kb wildtype and Pds5a KO mESC data created 741 in this study.

742 For pileup analysis at $\mathrm{HiC}$ loops we used coolpup.py ${ }^{95}$ and took averaged the calculated 743 observed over expected interactions within a $105 \mathrm{~kb} \times 105 \mathrm{~kb}$ window centered on the 744 loops at $5 \mathrm{~kb}$ resolution.

745 For calculation and visualization of compartment strengths, relative contact probabilities, 746 insulation scores and differential $\mathrm{HiC}$ contact matrices we used the R package GENOVA 747 (https://github.com/robinweide/GENOVA) ${ }^{96}$.

748 Insulation score analysis was conducted as previously described in $\left({ }^{97}\right.$.

749 Insulation scores ${ }^{60}$ were computed at $10 \mathrm{~kb}$ resolution using GENOVA. Bins with an 750 insulation score greater than -1 were excluded from the analysis. To qualify as 
751 differentially insulated bins, either wild type or Pds5a KO mESCs had to be lower than -

7520.2 to exclude very lowly insulated portions of the genome. Insulation gaining and losing

753 regions were defined as bins that had an absolute change in insulation between wild 754 type and Pds5a KO of 0.2 . 


\section{MAIN FIGURE TITLES AND LEGENDS}

756

757

758

759

760

761

762

763

764

765

766

767

768

769

770

771

772

773

774

775

776

777

778

779

780

781

782

783

Fig. 1. CRISPR screen of cPRC1-dependent gene silencing reveals PDS5A. a, Schematic of ectopic dual reporter locus consisting of 7x TetO landing sites flanked by an upstream Ef1a promoter driven BFP and a downstream PGK driven puromycin/GFP. Underneath shown are genomic ChIP-CapSeq screenshot of polycomb proteins and histone modifications before (black) and after TetR-Cbx7 expression (orange). b, Flow cytometry histograms of GFP reporter expression activity at continuous binding of TetRCbx7 fusion proteins (left) and after doxycyline induced release from the locus for 6 days (right). Top panel shows propagation of silencing after CRISPR-Cas9 infection utilizing a scrambled control sgRNA, and bottom panel CRISPR-Cas9 with sgRNA directed against Ring1b. Percentages refer to fraction of GFP-negative cells. c, Schematic of CRISPR screen design and flow cytometry sorting scheme. $\mathrm{MOI}=$ multiplicity of infection. Below, all nuclear factors genes targeted in the CRISPR screen (sgRNA library described in ${ }^{32}$ ) are ranked by their corresponding CRISPR significance score (-log 10 MAGeCK significance score ${ }^{32} ; \mathrm{n}=$ mean of three independent experiments)). d, Flow cytometry histograms of GFP reporter expression before and after doxycycline-induced release of TetR-Cbx7 binding for 4 days in wildtype reporter cells and FACS sorted Pds5a-sgRNA CRISPR-Cas9 targeted population. Percentages refer to fraction of GFP-negative cells.

\section{Fig. 2. Loss of cohesin regulator PDS5A results in de-repression of canonical} PRC1/PRC2 target genes. a, Western blot characterization of cohesin and polycomb proteins and histone modifcations in two independent Pds5a KO mESC cell lines and their corresponding wild types. b, Calibrated ChIP-seq heatmaps of PRC1 proteins (Ring1B, Pcgf1 (variant PRC1), Cbx7 (canonical PRC1)) and its histone modification H2AK119ub, as well as PRC2 proteins and histone modification Suz12 and H3K27me3, respectively. cChiP-seq signal is plotted around the TSS (+/- 5kb). Genes classified as PRC1/PRC2 shared genes (red; $n=2895$ ) yield a Bio-Cap peak (Data from ${ }^{55}$ ) for nonmethylated $\mathrm{CpG}$ island, Ring1b peak for PRC1 and a Suz12 peak for PRC2 within 3kb 
784

785

786

787

788

789

790

791

792

793

794

795

796

797

798

799

800

801

802

803

804

805

806

807

808

809

810

811

812

of the TSS. PcG vPRC1 classified genes (blue; $n=2448$ ) have only a Ring1b peak within $3 \mathrm{~kb}$ of the TSS, and genes with no Ring1b or Suz12 peak are fall in the class of non-PcG (yellow;n=10591). Genes without a non-methylated CpG island (NMI) are in class nonNMI ( $\mathrm{n}=7042)$. c, Volcano plot of RNA-seq expression change in Pds5a genetrap (GT) KO vs. wild type mESCs. Genes from the PRC1/PRC2 shared class $(n=2895)$ with increased or decreased expression are marked in red (padj $<0.05$ and log2 fold change $>0.5$ or $<-0.5)$. Total numbers of up-/and down-regulated genes are indicated in the top left and right corners (PRC1/PRC2 shared class in red and of all genes in black). d, Volcano plot of RNA-seq expression change in 48 hrs IAA-treated vs. untreated dSuz12 Pcgf1 KO mESCs. Genes from the PRC1/PRC2 shared class $(n=2895)$ with increased or decreased expression are marked in red (padj $<0.05$ and log2 fold change $>0.5$ or $<-$ 0.5). Total numbers of up-/and down-regulated genes are indicated in the top left and right corners (PRC1/PRC2 shared class in red and of all genes in black). e, Dotplots of log2 fold changes of selected representative genes from the PRC1/PRC2 shared genes class in both independent Pds5a KO mESC cell lines. f, Stacked bargraphs of percent gene distribution between classes of all genes (left) and of up-regulated genes in both Pds5a KO cell lines, dSuz12 Pcgf1 KO IAA treated cells and in the overlap of Pds5a KO (GT) and dSuz12 Pcgf1 KO. g, Euler diagrams of overlap of all, as well as only PRC1/PRC2 shared up-regulated genes between both Pds5a KO cell lines (top) and between Pds5a KO (GT) and dSuz12 Pcgf1 KO. (H) Boxplots of RNA-seq log2 fold change expression changes for each gene class for both Pds5a KO mESC cell lines.

Fig. 3. PDS5A deletion has minimal effect on Polycomb repressive chromatin domains. a, Wildtype and Pds5a KO cChIP-seq heatmaps of PRC1 (Ring1b and H2AK119ub) and PRC2 (Suz12 and H3K27me3). cChiP-seq signal is plotted around the TSS (+/- 5kb). Heatmap is divided in 3 gene classes (see Fig2b): PRC1/PRC2 shared genes (red; $n=2895$ ), PcG vPRC1 (blue; $n=2448$ ), non-PcG (yellow; $n=10591$ ). b, cChIPseq heatmaps of PRC1 (Ring1b and H2AK119ub) in 48 hrs IAA-treated vs. untreated dSuz12 Pcgf1 KO mESCs. c, Wild type and Pds5a KO scatterplots of cChIP signal 
813 around TSS for each gene (Sum of TSS +/- $2.5 \mathrm{~kb}$ ). Genes from the PRC1/PRC2 shared 814 gene class, which are up-regulated in Pds5a KO cells (see Fig 2c) are marked in red and 815 selected representative genes are labelled individually (see Fig 2d). d, cChIP scatterplots 816 as in (c) for 48 hrs IAA-treated vs. untreated dSuz12 Pcgf1 KO mESCs. e, IGV genomic 817 cChIP-seq track screenshot of RNA-seq, PRC1 and PRC2 polycomb proteins and 818 histone modifications in wildtype (black) and Pds5a KO (red) mESCs at the Otx1 locus 819 and f, at the Six1 locus. g, ATAC-seq heatmap, boxplots for each gene class (see Fig. 3a) and the corresponding subset of transcriptionally up-regulated genes.

Fig. 4. PDS5A deletion increases cohesin-dependent loop extrusion causing TAD

823 boundary violations. a, KR normalized $\mathrm{Hi}-\mathrm{C}$ contact matrices of chromosome 2 in wild 824 type (left) and Pds5a KO (right) mESCs. b, Eigenvector compartment signal tracks at 250 $825 \mathrm{~kb}$ bin resolution are plotted for both wild type and PDS5A. c, Wild type vs. Pds5a KO 826 compartment strength (generated in GENOVA) are plotted. One dot for each

827 chromosome. d, Aggregate Hi-C pileup analysis (observed/expected enrichment) using 828 coolpup.py from wild type (top panel) and Pds5a KO (bottom panel) at wild type mESC TADs called in Bonev et al. $2017^{59}$ mESC dataset (5kb resolution). e, Aggregate Hi-C pileup analysis (observed/expected enrichment) using coolpup.py from wild type (left panels) and Pds5a KO (right panels) at wild type mESC loops +/- 100kb called in Bonev et al. $2017^{59} \mathrm{mESC}$ dataset (5kb resolution). Bottom panels show analysis for subset of only Cohesin dependent (CTCF anchored) and PRC1 dependent (Ring1b anchored)

834 loops. In each pileup plot, the mean enrichment scores of the three center pixels is 835 displayed in the top left corner. f, RCP plot (Relative contact probability) depicting 836 genomic distance dependent contact frequency for wild type and Pds5a KO mESCs. 837 Dashed line indicates expected size range of TADs and compartments. (1) Relative 838 reduction of short-range contacts (0-500kb) in Pds5a KO; (2) Relative increase of mid839 range contacts (500kb-5mb) in Pds5a KO; (3) Relative reduction of long-range contacts 840 (>5mb) in Pds5a KO. g, Quantification of TAD border violation. Boxplot of log2 change 841 in $\mathrm{HiC}$ contacts with $\mathrm{n}+(\mathrm{x}$-axis) neighboring TADs between wild type and Pds5a KO. 
843 Fig. 5. PDS5A is required for maintenance of repressive polycomb loops crossing

844 ultra-Iong distances. a, Loop pileup analysis of $\mathrm{HiC}$ data of Bonev et al. $2017^{59} \mathrm{mESCs}$, wildtype and Pds5a KO at not PcG loops, PcG loops (between shared PRC1/2 and/or VPRC1 genes) and shared PRC1/PRC2 PcG loops (between shared PRC1/2 genes). b, Loop pileup analysis of $\mathrm{HiC}$ data of Bonev et al. $2017^{59} \mathrm{mESCs}$, wildtype and Pds5a KO at shared PRC1/PRC2 PcG loops with left and right anchor not-upregulated in Pds5a $\mathrm{KO}$ and at shared PRC1/PRC2 PcG loops with left anchor not-upregulated in Pds5a KO and right anchor upregulated in Pds5a KO. c, cChIP-seq profile plots of PRC1 (Ring1b upregulated PRC1/PRC2 shared target genes (left and right loop anchors; $n=411$ ) and loops between upregulated PRC1/PRC2 shared target genes (left loop anchor represents the not upregulated side and right loop anchor the Pds5a KO transcriptionally upregulated side; $n=65)$. d, Cumulative distribution plots of loop lengths corresponding to loop classes shown in Fig $5 \mathrm{~A}$ and $5 \mathrm{~B}$ as well as e, number of TADs within the $A$ and B compartment traversed by loops of each class.

Fig. 6. Loss of repressive Polycomb loops is linked to cohesin-mediated insulation (+/- 300kb) in wild type and Pds5a KO. Left panel shows bins with unchanged insulation ( $n=40838)$; Right panel shows insulation gaining bins $(n=1408$; minimum insulation score decrease of -0.2). b, Boxplots for each gene class showing genomic distance $(\mathrm{kb})$ to the closest insulation gaining $10 \mathrm{~kb}$ bin of the TSS of each gene within its gene class. c, Loss of 3D interaction of Dlx1/2 with HoxD cluster. Shown are wild type and Pds5a KO HiC matrices and a differential HiC matrix (blue = loss; red = gain of contacts in Pds5a $\mathrm{KO}$;

867 10kb resolution and ICE-normalized). Virtual 4C tracks for both wt and Pds5a KO from 868 indicated viewpoints v1-3 are plotted below. Genomic screenshots illustrating polycomb 869 group proteins and histone modification cChIP at DIx1/2 and the HoxD cluster. Insulation 
bioRxiv preprint doi: https://doi.org/10.1101/2021.12.15.472841; this version posted December 16, 2021. The copyright holder for this preprint (which was not certified by peer review) is the author/funder. All rights reserved. No reuse allowed without permission.

871 the Insulation gaining region (indicated by black bar). Change in RNA-seq signal for DIx2

872 is plotted underneath. 


\section{EXTENDED DATA FIGURE TITLES AND LEGENDS}

874

875

876

877

878

879

880

881

882

883

884

885

886

887

888

889

890

891

892

893

894

895

896

897

898

899

900

901

\section{Extended Data Fig. 1. CRISPR screen of cPRC1-dependent gene silencing reveals}

PDS5A. a, Genomic ChIP-CapSeq screenshot of polycomb proteins and histone modifications before (black) and after TetR-Cbx7 expression (orange) at HoxD cluster. b, Flow cytometry scatterplots of CRISPR screen sorting scheme to enrich for GFPpositive reporter mESCs. GFP expression of reporter is shown on x-axis and TetR-Cbx7 expression represented by m-cherry on y-axis. Sorting gates applied are indicated in each plot. c, Western blots for RING1B and PDS5A of wild type TetR-Cbx7 mESC reporter cell lines and Ring1b KO (left panel) and Pds5a KO (right panel) clonal cell lines.

\section{Extended Data Fig. 2. Pds5a KO does not impair mESC stem cell markers or cell} cycle profiles and causes de-repression of canonical PRC1/PRC2 target genes in second independent Pds5a KO cell line. a, Alkaline phosphatase staining of wildtype and Pds5a KO mESCs. b, Flow cytometry histogram after Hoechst staining showing cell cycle distribution plot of G1, S and G2M phase in wild type and Pds5a KO. c, Flow cytometry scatterplots of stem cell marker staining (SSEA1, OCT3/4 and SOX2) including corresponding antibody isotype controls (right panels). d, Volcano plot of RNA-seq expression change in PDS5A CRISPR-Cas9 KO vs. wild type mESCs (Pds5a KO/WT). Genes from the PRC1/PRC2 shared class $(n=2895)$ with increased or decreased expression are marked in red (padj $<0.05$ and log2 fold change $>0.5$ or $<-0.5$ ). Total numbers of up-/and down-regulated genes are indicated in the top left and right corners (PRC1/PRC2 shared class in red and of all genes in black). e, Gene ontology analysis plots of enriched gene groups among all up-regulated genes (padj < 0.05 and log2 fold change > 0.5) in PDS5A genetrap KO mESCs and f, PDS5A CRISPR-Cas9 KO mESCs. g, Heatmap of log2 fold changes in RNA-seq of all de-regulated genes (padj $<0.05$ and log2 fold change $>0.5$ or $<-0.5$ ) upon PDS5A loss for PDS5A genetrap (GT) and PDS5A CRISPR-Cas9 KO mESCs. h, Stacked bargraphs of percent gene distribution of downregulated genes in both Pds5a KO cell lines individually and in their overlap. i, Euler 
902 diagrams of overlap of all (top), as well as only PRC1/PRC2 shared down-regulated 903 genes (bottom) between both Pds5a KO cell lines.

904

905

Extended Data Fig. 3. PDS5A deletion has minimal effect on Polycomb repressive

906 chromatin domains. a, Profile plots quantifying mean cChIP signal for each IP (Ring1b, 907 H2AK119ub, Suz12, H3K27me3) and gene class in Fig 3A for wildtype and Pds5a KO 908 mESCs and b, 48 hrs IAA-treated vs. untreated dSuz12 Pcgf1 KO mESCs. c, 909 Scatterplots of cChIP signal around TSS for each gene (Sum of TSS +/- $2.5 \mathrm{~kb}$ ). Genes 910 from the PRC1/PRC2 shared gene class are colored in red and selected representative 911 genes are labeled individually (see Fig 2d) for wildtype and Pds5a KO mESCs and d, 48 912 hrs IAA-treated vs. untreated dSuz12 Pcgf1 KO mESCs. e, Boxplots of PRC1 and PRC2 913 complex and histone modification cChIP signal within the entire PRC1/PRC2 shared or 914 VPRC1 only gene class and their corresponding subsets of transcriptionally up-regulated 915 genes for 48 hrs IAA-treated vs. untreated dSuz12 Pcgf1 KO mESCs and f, wildtype and 916 Pds5a KO mESCs.

Extended Data Fig. 4. PDS5A colocalizes with cohesin and stabilizes chromatin binding. a, Heatmaps of compartment signal at $250 \mathrm{~kb}$ resolution for chromosomes 1 and 2 in wildtype and Pds5a KO. b, Scatterplot of compartment signal (Eigenvector) for each $250 \mathrm{~kb}$ bin in the genome in wildtype vs. Pds5a KO. c, Cumulative distributions of called TAD domains in wildtype and Pds5a KO. d, Wildtype and Pds5a KO cChIP-seq heatmaps of Rad21, PDS5A and CTCF. cChIP-seq signal is plotted around common wild type/KO ( $n=15941)$, wt only $(n=4288)$ or Pds5a KO only $(n=6789)$ peak centers +- 5kb. e,

926 Euler diagrams of overlap of wild type CTCF, Rad21 and PDS5A peaks (left) and overlap 927 of wild type and Pds5a KO called Rad21 peaks. f, Profile plots quantifying mean cChIP 928 signal of Rad21 and PDS5A cChIP at Rad21 peaks. g, IGV genomic cChIP-seq 929 screenshot illustrating CTCF (wild type only), Rad21 and PDS5A in wildtype and Pds5a $930 \mathrm{KO}$. 
932 Extended Data Fig. 5. PDS5A is required for maintenance of repressive polycomb

933 loops crossing ultra-long distances. a, cChIP-seq heatmaps of PRC1 (Ring1b and

$934 \mathrm{H} 2 \mathrm{AK} 119 \mathrm{ub})$ and PRC2 (Suz12 and H3K27me3) at Polycomb loops between shared

935 PRC1/PRC2 genes. Heatmap is divided in loops between not-upregulated PRC1/PRC2

936 shared target genes (left and right loop anchors; $n=411$ ) and loops between upregulated

937 PRC1/PRC2 shared target genes (left loop anchor represents the not upregulated side

938 and right loop anchor the Pds5a KO transcriptionally upregulated side; $\mathrm{n}=65$ ).

939

940

Extended Data Fig. 6. Loss of repressive Polycomb loops is linked to cohesin-

941 mediated insulation gain. a, Heatmaps and average profile plots of insulation score of

942 genomic $10 \mathrm{~kb}$ bins (+/- 300kb) in wild type and Pds5a KO. Shown are insulation losing

943 bins ( $n=17269$; minimum insulation score increase of 0.2). b, Wild type and Pds5a KO

$944 \mathrm{HiC}$ matrices and a differential $\mathrm{HiC}$ matrix (blue = loss; red = gain of contacts in Pds5a

$945 \mathrm{KO}$; 10kb resolution and ICE-normalized). Genomic screenshots of polycomb group 946 proteins and histone modification cChIP are displayed for the HoxB cluster and Ppp1r1b

947 . Insulation scores, as well as cChIP IGV screenshots of cohesin (Rad21) and CTCF are 948 shown at the Insulation gaining region (indicated by black bar). Change in RNA-seq 949 signal for DIx2 is plotted underneath. 
950

951

952

953

954

955

956

957

958

959

960

961

962

963

964

965

966

967

968

969

970

\section{REFERENCES}

1. Kassis, J. A., Kennison, J. A. \& Tamkun, J. W. Polycomb and Trithorax Group Genes in Drosophila. Genetics 206, 1699-1725 (2017).

2. Schuettengruber, B., Bourbon, H.-M., Croce, L. D. \& Cavalli, G. Genome Regulation by Polycomb and Trithorax: 70 Years and Counting. Cell 171, 34-57 (2017).

3. Béguelin, W. et al. EZH2 is required for germinal center formation and somatic EZH2 mutations promote lymphoid transformation. Cancer Cell 23, 677-692 (2013).

4. Béguelin, W. et al. EZH2 and BCL6 Cooperate to Assemble CBX8-BCOR Complex to Repress Bivalent Promoters, Mediate Germinal Center Formation and Lymphomagenesis. Cancer Cell 30, 197-213 (2016).

5. Chan, H. L. et al. Polycomb complexes associate with enhancers and promote oncogenic transcriptional programs in cancer through multiple mechanisms. Nat Commun 9, 3377 (2018).

6. Donaldson-Collier, M. C. et al. EZH2 oncogenic mutations drive epigenetic, transcriptional, and structural changes within chromatin domains. Nat Genet 51, 517-528 (2019).

7. Duan, R., Du, W. \& Guo, W. EZH2: a novel target for cancer treatment. J Hematol Oncol 13, 104 (2020).

8. Piunti, A. \& Shilatifard, A. The roles of Polycomb repressive complexes in mammalian development and cancer. Nat Rev Mol Cell Biol 22, 326-345 (2021).

9. Schlesinger, Y. et al. Polycomb-mediated methylation on Lys 27 of histone H3 pre-marks genes for de novo methylation in cancer. Nat Genet 39, 232-236 (2007). 
971 10. Yap, D. B. et al. Somatic mutations at EZH2 Y641 act dominantly through a mechanism of

972 selectively altered PRC2 catalytic activity, to increase H3K27 trimethylation. Blood 117,

$973 \quad 2451-2459$ (2011).

974 11. Cao, R. et al. Role of histone H3 lysine 27 methylation in Polycomb-group silencing. Science

$975 \quad 298,1039-1043$ (2002).

976 12. Eskeland, R. et al. Ring1B Compacts Chromatin Structure and Represses Gene Expression

977 Independent of Histone Ubiquitination. Mol Cell 38, 452-464 (2010).

978 13. Gao, Z. et al. PCGF homologs, CBX proteins, and RYBP define functionally distinct PRC1

979 family complexes. Mol Cell 45, 344-356 (2012).

980 14. Wang, H. et al. Role of histone H2A ubiquitination in Polycomb silencing. Nature 431, 873-

981878 (2004).

982 15. Bernstein, B. E. et al. A bivalent chromatin structure marks key developmental genes in

983 embryonic stem cells. Cell 125, 315-326 (2006).

984 16. Wang, L. et al. Hierarchical Recruitment of Polycomb Group Silencing Complexes. Molecular

985 Cell 14, 637-646 (2004).

986 17. Blackledge, N. P. \& Klose, R. J. The molecular principles of gene regulation by Polycomb

987 repressive complexes. Nat Rev Mol Cell Biol 1-19 (2021) doi:10.1038/s41580-021-00398-y.

988 18. Blackledge, N. P. et al. PRC1 Catalytic Activity Is Central to Polycomb System Function.

$989 \quad$ Molecular Cell 77, 857-874.e9 (2020).

990 19. Fursova, N. A. et al. Synergy between Variant PRC1 Complexes Defines Polycomb-Mediated

991 Gene Repression. Mol Cell 74, 1020-1036.e8 (2019). 
20. Kasinath, V. et al. JARID2 and AEBP2 regulate PRC2 in the presence of H2AK119ub1 and other histone modifications. Science 371, eabc3393 (2021).

21. Bantignies, F. et al. Polycomb-dependent regulatory contacts between distant Hox loci in Drosophila. Cell 144, 214-226 (2011).

22. Boyle, S. et al. A central role for canonical PRC1 in shaping the 3D nuclear landscape. Genes Dev. (2020) doi:10.1101/gad.336487.120.

23. Eagen, K. P., Aiden, E. L. \& Kornberg, R. D. Polycomb-mediated chromatin loops revealed by a subkilobase-resolution chromatin interaction map. PNAS 114, 8764-8769 (2017).

24. Isono, K. et al. SAM domain polymerization links subnuclear clustering of PRC1 to gene silencing. Dev Cell 26, 565-577 (2013).

25. Kundu, S. et al. Polycomb Repressive Complex 1 Generates Discrete Compacted Domains that Change during Differentiation. Mol Cell 65, 432-446.e5 (2017).

26. Rhodes, J. D. P. et al. Cohesin Disrupts Polycomb-Dependent Chromosome Interactions in Embryonic Stem Cells. Cell Reports 30, 820-835.e10 (2020).

27. Schoenfelder, S. et al. Polycomb repressive complex PRC1 spatially constrains the mouse embryonic stem cell genome. Nat Genet 47, 1179-1186 (2015).

1008 28. Ogiyama, Y., Schuettengruber, B., Papadopoulos, G. L., Chang, J.-M. \& Cavalli, G. Polycomb1009 Dependent Chromatin Looping Contributes to Gene Silencing during Drosophila 1010 Development. Mol Cell 71, 73-88.e5 (2018).

1011 29. Scelfo, A. et al. Functional Landscape of PCGF Proteins Reveals Both RING1A/B-Dependent1012 and RING1A/B-Independent-Specific Activities. Molecular Cell 74, 1037-1052.e7 (2019). 
1013 30. Zepeda-Martinez, J. A. et al. Parallel PRC2/cPRC1 and vPRC1 pathways silence lineage-

1014 specific genes and maintain self-renewal in mouse embryonic stem cells. Sci Adv 6,

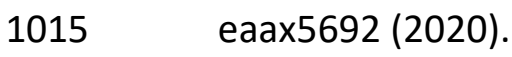

1016 31. Moussa, H. F. et al. Canonical PRC1 controls sequence-independent propagation of

1017 Polycomb-mediated gene silencing. Nat Commun 10, 1931 (2019).

1018 32. Michlits, G. et al. CRISPR-UMI: single-cell lineage tracing of pooled CRISPR-Cas9 screens. Nat

$1019 \quad$ Methods 14, 1191-1197 (2017).

1020 33. Li, W. et al. MAGeCK enables robust identification of essential genes from genome-scale

1021 CRISPR/Cas9 knockout screens. Genome Biol 15, 554 (2014).

1022 34. Haering, C. H., Farcas, A.-M., Arumugam, P., Metson, J. \& Nasmyth, K. The cohesin ring

1023 concatenates sister DNA molecules. Nature 454, 297-301 (2008).

1024 35. Arruda, N. L. et al. Distinct and overlapping roles of STAG1 and STAG2 in cohesin localization

1025 and gene expression in embryonic stem cells. Epigenetics \& Chromatin 13, 32 (2020).

1026 36. Gandhi, R., Gillespie, P. J. \& Hirano, T. Human Wapl is a cohesin-binding protein that

1027 promotes sister-chromatid resolution in mitotic prophase. Curr Biol 16, 2406-2417 (2006).

1028 37. Haarhuis, J. H. I. et al. The Cohesin Release Factor WAPL Restricts Chromatin Loop

1029 Extension. Cell 169, 693-707.e14 (2017).

1030 38. Huis in 't Veld, P. J. et al. Characterization of a DNA exit gate in the human cohesin ring.

$1031 \quad$ Science 346, 968-972 (2014).

1032 39. Kueng, S. et al. Wapl controls the dynamic association of cohesin with chromatin. Cell 127,

1033 955-967 (2006). 
1034 40. Losada, A., Yokochi, T., Kobayashi, R. \& Hirano, T. Identification and characterization of 1035 SA/Scc3p subunits in the Xenopus and human cohesin complexes. J Cell Biol 150, 405-416 1036 (2000).

1037 41. Tedeschi, A. et al. Wapl is an essential regulator of chromatin structure and chromosome 1038 segregation. Nature 501, 564-568 (2013).

1039 42. Wutz, G. et al. Topologically associating domains and chromatin loops depend on cohesin 1040 and are regulated by CTCF, WAPL, and PDS5 proteins. EMBO J 36, 3573-3599 (2017).

1041 43. Zhang, N. et al. Characterization of the interaction between the cohesin subunits Rad21 and 1042 SA1/2. PLoS One 8, e69458 (2013).

1043 44. Cuadrado, A. et al. Specific Contributions of Cohesin-SA1 and Cohesin-SA2 to TADs and 1044 Polycomb Domains in Embryonic Stem Cells. Cell Reports 27, 3500-3510.e4 (2019).

1045 45. Elling, U. et al. A reversible haploid mouse embryonic stem cell biobank resource for 1046 functional genomics. Nature 550, 114-118 (2017).

1047 46. Kagey, M. H. et al. Mediator and cohesin connect gene expression and chromatin 1048 architecture. Nature 467, 430-435 (2010).

1049 47. Lavagnolli, T. et al. Initiation and maintenance of pluripotency gene expression in the 1050 absence of cohesin. Genes Dev. 29, 23-38 (2015).

1051 48. Nitzsche, A. et al. RAD21 cooperates with pluripotency transcription factors in the 1052 maintenance of embryonic stem cell identity. PLoS One 6, e19470 (2011).

1053 49. Chen, S., Jiao, L., Liu, X., Yang, X. \& Liu, X. A Dimeric Structural Scaffold for PRC2-PCL 1054 Targeting to CpG Island Chromatin. Mol Cell 77, 1265-1278.e7 (2020). 
1055

1056

1057

1058

1059

1060

1061

1062

1063

1064

1065

1066

1067

1068

1069

1070

1071

1072

1073

1074

1075

50. Deaton, A. M. \& Bird, A. CpG islands and the regulation of transcription. Genes Dev. 25, 1010-1022 (2011).

51. Farcas, A. M. et al. KDM2B links the Polycomb Repressive Complex 1 (PRC1) to recognition of CpG islands. Elife 1, e00205 (2012).

52. He, J. et al. Kdm2b maintains murine embryonic stem cell status by recruiting PRC1 complex to CpG islands of developmental genes. Nat Cell Biol 15, 373-384 (2013).

53. Li, H. et al. Polycomb-like proteins link the PRC2 complex to CpG islands. Nature 549, 287291 (2017).

54. Wu, X., Johansen, J. V. \& Helin, K. Fbxl10/Kdm2b recruits polycomb repressive complex 1 to CpG islands and regulates H2A ubiquitylation. Mol Cell 49, 1134-1146 (2013).

55. Long, H. K. et al. Epigenetic conservation at gene regulatory elements revealed by nonmethylated DNA profiling in seven vertebrates. eLife 2, e00348 (2013).

56. Busslinger, G. A. et al. Cohesin is positioned in mammalian genomes by transcription, CTCF and Wapl. Nature 544, 503-507 (2017).

57. Rao, S. S. P. et al. Cohesin Loss Eliminates All Loop Domains. Cell 171, 305-320.e24 (2017).

58. Knight, P. A. \& Ruiz, D. A fast algorithm for matrix balancing. IMA Journal of Numerical Analysis 33, 1029-1047 (2013).

59. Bonev, B. et al. Multiscale 3D Genome Rewiring during Mouse Neural Development. Cell 171, 557-572.e24 (2017).

60. Crane, E. et al. Condensin-Driven Remodeling of X-Chromosome Topology during Dosage Compensation. Nature 523, 240-244 (2015). 
1076

1077

1078

1079

1080

1081

1082

1083

1084

1085

1086

1087

1088

1089

1090

1091

1092

1093

1094

1095

1096

1097
61. Cunningham, M. D. et al. Wapl antagonizes cohesin binding and promotes Polycomb-group silencing in Drosophila. Development 139, 4172-4179 (2012).

62. Zhang, B. et al. Dosage Effects of Cohesin Regulatory Factor PDS5 on Mammalian Development: Implications for Cohesinopathies. PLoS One 4, e5232 (2009).

63. Katoh-Fukui, Y. et al. Male-to-female sex reversal in M33 mutant mice. Nature 393, 688692 (1998).

64. Lau, M. S. et al. Mutation of a nucleosome compaction region disrupts Polycomb-mediated axial patterning. Science 355, 1081-1084 (2017).

65. Cohen, I. et al. PRC1 preserves epidermal tissue integrity independently of PRC2. Genes Dev. 33, 55-60 (2019).

66. Pasini, D., Bracken, A. P., Hansen, J. B., Capillo, M. \& Helin, K. The Polycomb Group Protein Suz12 Is Required for Embryonic Stem Cell Differentiation. Molecular and Cellular Biology 27, 3769-3779 (2007).

67. Riising, E. M. et al. Gene silencing triggers polycomb repressive complex 2 recruitment to CpG islands genome wide. Mol Cell 55, 347-360 (2014).

68. Akasaka, T. et al. Mice doubly deficient for the Polycomb Group genes Mel18 and Bmi1 reveal synergy and requirement for maintenance but not initiation of Hox gene expression. Development 128, 1587-1597 (2001).

69. Hansen, K. H. et al. A model for transmission of the H3K27me3 epigenetic mark. Nat Cell Biol 10, 1291-1300 (2008).

70. Chambeyron, S. \& Bickmore, W. A. Chromatin decondensation and nuclear reorganization of the HoxB locus upon induction of transcription. Genes Dev 18, 1119-1130 (2004). 
1098

1099

1100

1101

1102

1103

1104

1105

1106

1107

1108

1109

1110

1111

1112

1113

1114

1115

1116

1117

1118

71. Illingworth, R. S. Chromatin folding and nuclear architecture: PRC1 function in 3D. Curr Opin Genet Dev 55, 82-90 (2019).

72. Adane, B. et al. STAG2 loss rewires oncogenic and developmental programs to promote metastasis in Ewing sarcoma. Cancer Cell 39, 827-844.e10 (2021).

73. Kubo, N. et al. Promoter-proximal CTCF binding promotes distal enhancer-dependent gene activation. Nat Struct Mol Biol 28, 152-161 (2021).

74. Nora, E. P. et al. Targeted Degradation of CTCF Decouples Local Insulation of Chromosome Domains from Genomic Compartmentalization. Cell 169, 930-944.e22 (2017).

75. Rowley, M. J. \& Corces, V. G. Organizational principles of 3D genome architecture. Nat Rev Genet 19, 789-800 (2018).

76. Schwarzer, W. et al. Two independent modes of chromatin organization revealed by cohesin removal. Nature 551, 51-56 (2017).

77. Rao, S. S. P. et al. A 3D Map of the Human Genome at Kilobase Resolution Reveals Principles of Chromatin Looping. Cell 159, 1665-1680 (2014).

78. Dobin, A. et al. STAR: ultrafast universal RNA-seq aligner. Bioinformatics 29, 15-21 (2013).

79. Anders, S., Pyl, P. T. \& Huber, W. HTSeq--a Python framework to work with high-throughput sequencing data. Bioinformatics 31, 166-169 (2015).

80. Love, M. I., Huber, W. \& Anders, S. Moderated estimation of fold change and dispersion for RNA-seq data with DESeq2. Genome Biol 15, 550 (2014).

81. Zhu, A., Ibrahim, J. G. \& Love, M. I. Heavy-tailed prior distributions for sequence count data: removing the noise and preserving large differences. Bioinformatics 35, 2084-2092 (2019). 
1119 82. $\mathrm{Wu}, \mathrm{T}$. et al. clusterProfiler 4.0: A universal enrichment tool for interpreting omics data. The

$1120 \quad$ Innovation 2, 100141 (2021).

1121 83. Yu, G., Wang, L.-G., Han, Y. \& He, Q.-Y. clusterProfiler: an R Package for Comparing

1122 Biological Themes Among Gene Clusters. OMICS: A Journal of Integrative Biology 16, 284-

$1123287(2012)$.

1124 84. Li, H. et al. The Sequence Alignment/Map format and SAMtools. Bioinformatics 25, 2078-

11252079 (2009).

1126 85. Bonhoure, N. et al. Quantifying ChIP-seq data: a spiking method providing an internal

1127 reference for sample-to-sample normalization. Genome Res 24, 1157-1168 (2014).

1128 86. Hu, B. et al. Biological chromodynamics: a general method for measuring protein occupancy

1129 across the genome by calibrating ChIP-seq. Nucleic Acids Res 43, e132 (2015).

1130 87. Ramírez, F. et al. deepTools2: a next generation web server for deep-sequencing data

$1131 \quad$ analysis. Nucleic Acids Res 44, W160-W165 (2016).

1132 88. Zhang, Y. et al. Model-based Analysis of ChIP-Seq (MACS). Genome Biology 9, R137 (2008).

1133 89. Servant, N. et al. HiC-Pro: an optimized and flexible pipeline for Hi-C data processing.

1134 Genome Biology 16, 259 (2015).

1135 90. Langmead, B. \& Salzberg, S. L. Fast gapped-read alignment with Bowtie 2. Nat Methods 9, 1136 357-359 (2012).

1137 91. Ramírez, F. et al. High-resolution TADs reveal DNA sequences underlying genome 1138 organization in flies. Nat Commun 9, 189 (2018).

1139 92. Wolff, J. et al. Galaxy HiCExplorer: a web server for reproducible Hi-C data analysis, quality $1140 \quad$ control and visualization. Nucleic Acids Research 46, W11-W16 (2018). 
1141 93. Wolff, J. et al. Galaxy HiCExplorer 3: a web server for reproducible Hi-C, capture Hi-C and

1142 single-cell Hi-C data analysis, quality control and visualization. Nucleic Acids Research 48,

1143 W177-W184 (2020).

1144 94. Durand, N. C. et al. Juicer Provides a One-Click System for Analyzing Loop-Resolution Hi-C

$1145 \quad$ Experiments. cels 3, 95-98 (2016).

1146 95. Flyamer, I. M., Illingworth, R. S. \& Bickmore, W. A. Coolpup.py: versatile pile-up analysis of

1147 Hi-C data. Bioinformatics 36, 2980-2985 (2020).

1148 96. Weide, R. H. van der et al. Hi-C Analyses with GENOVA: a case study with cohesin variants.

1149 2021.01.22.427620 https://www.biorxiv.org/content/10.1101/2021.01.22.427620v1 (2021)

$1150 \quad$ doi:10.1101/2021.01.22.427620.

1151 97. Kaaij, L. J. T., Mohn, F., van der Weide, R. H., de Wit, E. \& Bühler, M. The ChAHP Complex

1152 Counteracts Chromatin Looping at CTCF Sites that Emerged from SINE Expansions in Mouse.

$1153 \quad$ Cell 178, 1437-1451.e14 (2019). 
Fig.1

Bsteh et al.,

a
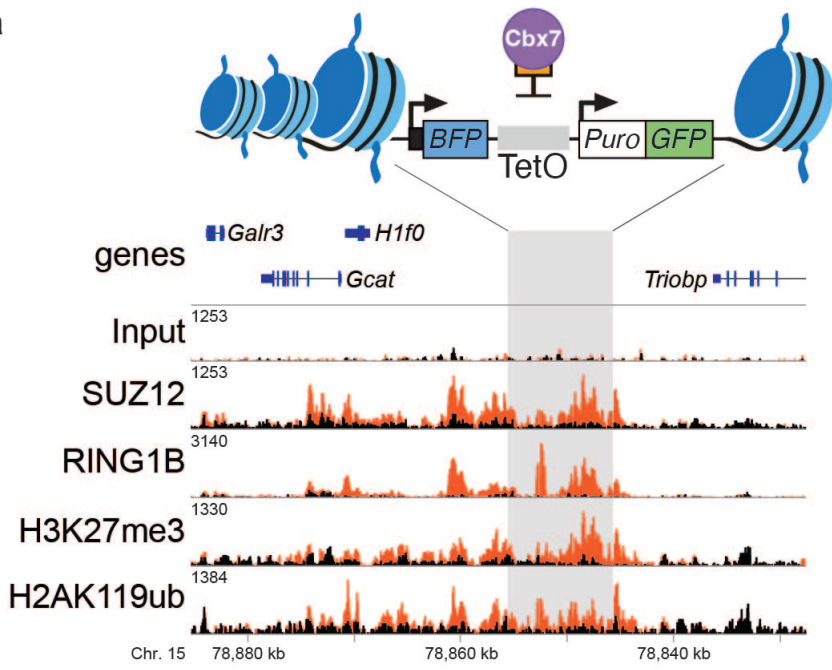

c

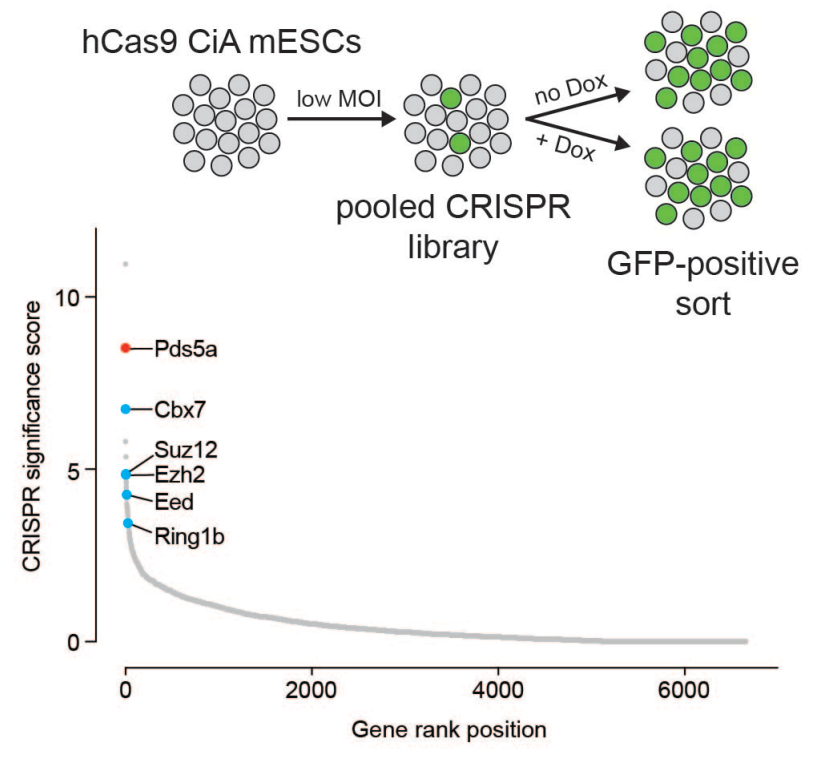

b
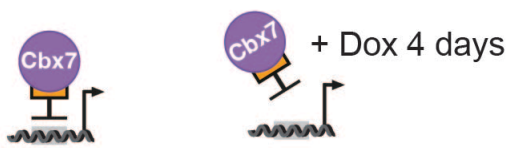

scramble

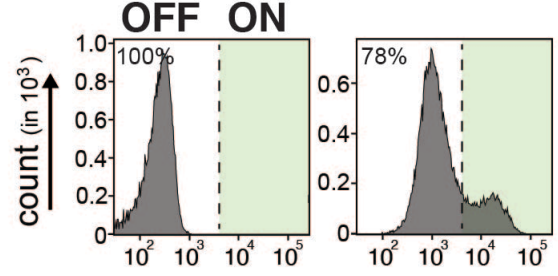

Ring 1b

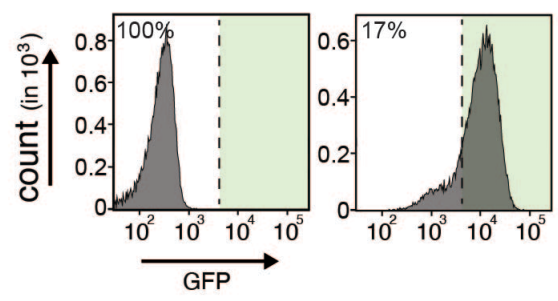

d
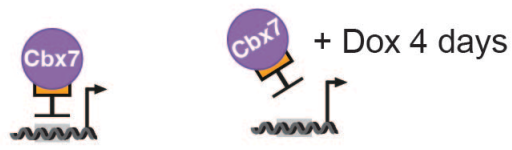

wildtype
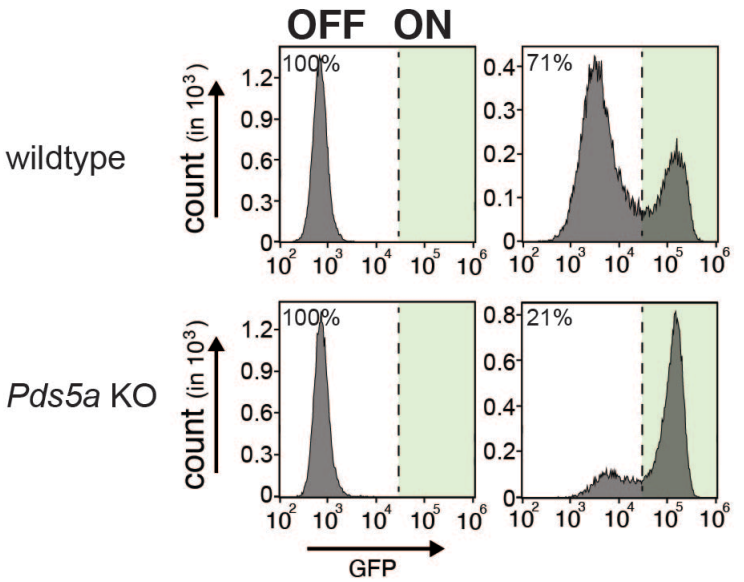
bioRxiv preprint doi: https://doi.org/10.1101/2021.12.15.472841; this version posted December 16, 2021. The copyright holder for this preprint (which was not certified by peer review) is the author/funder. All rights reserved. No reuse allowed without permission.

Fig.2
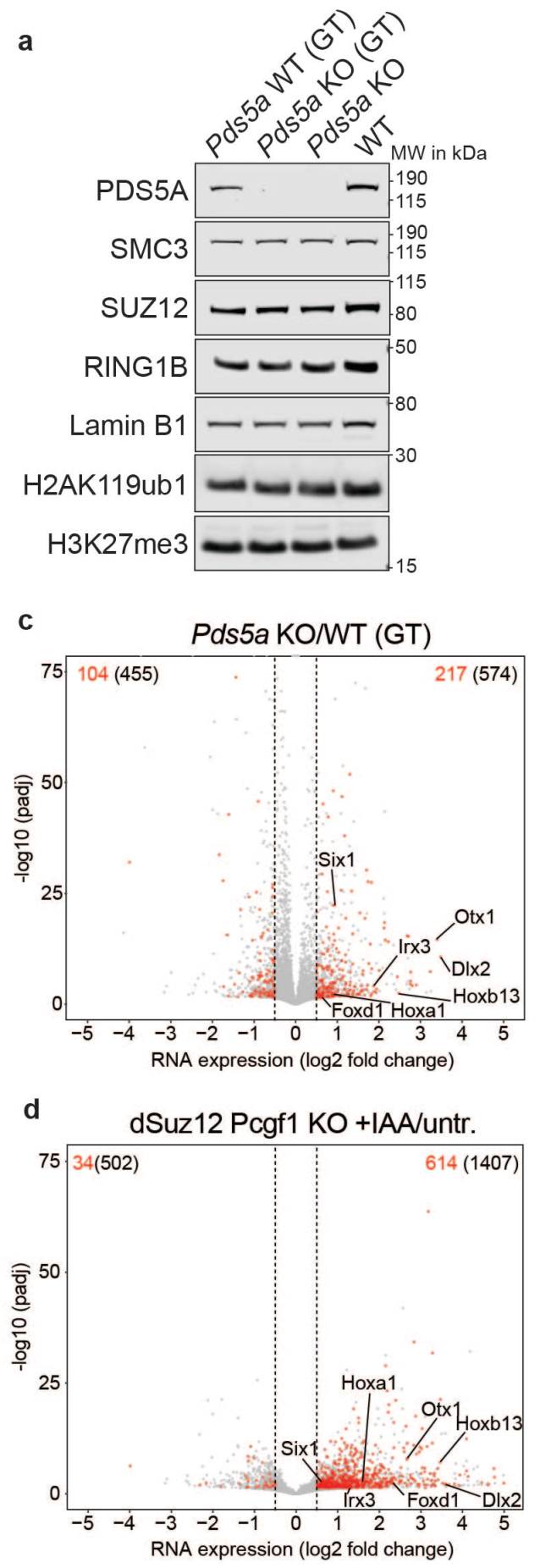

g
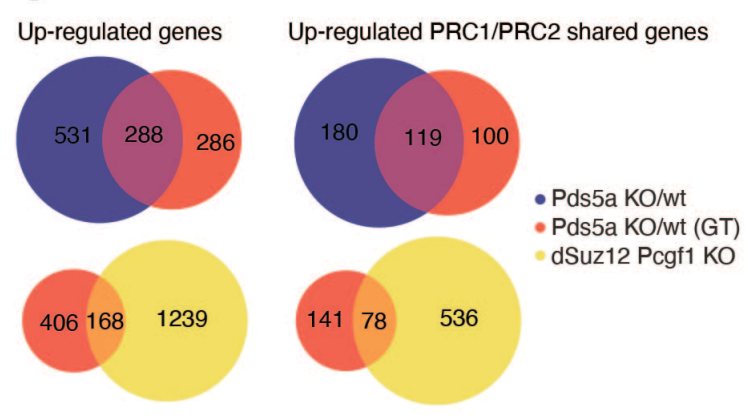

PRC1

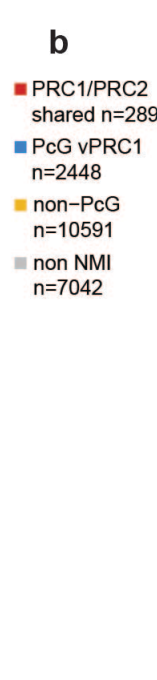

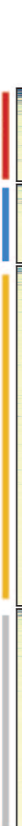

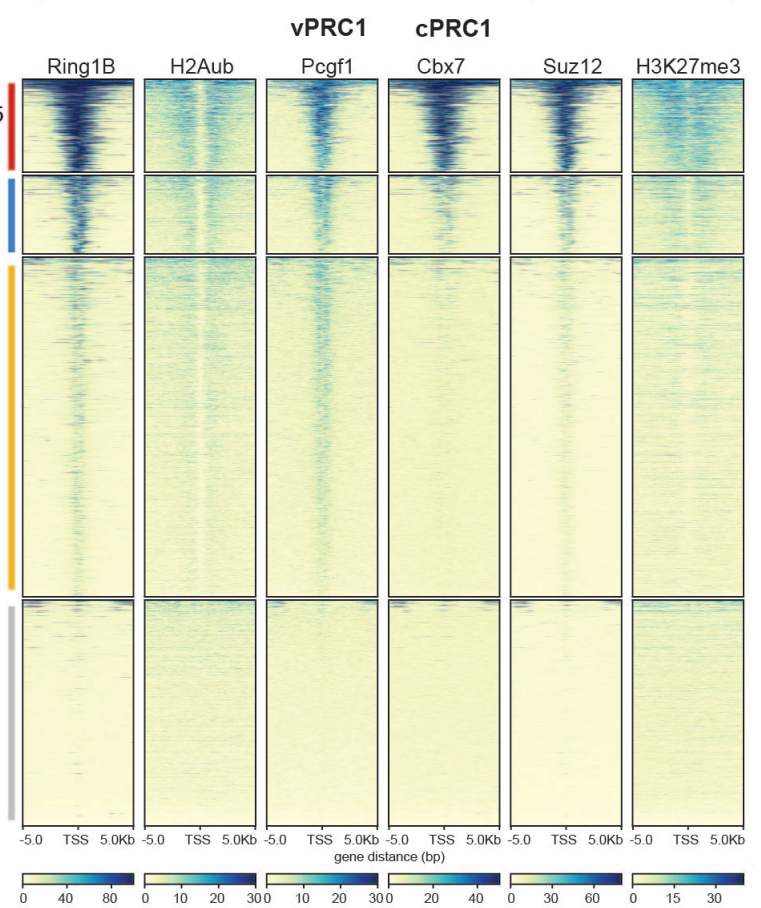

e

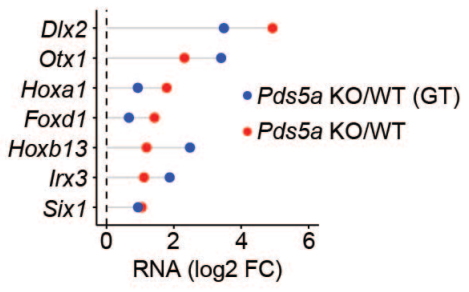

f

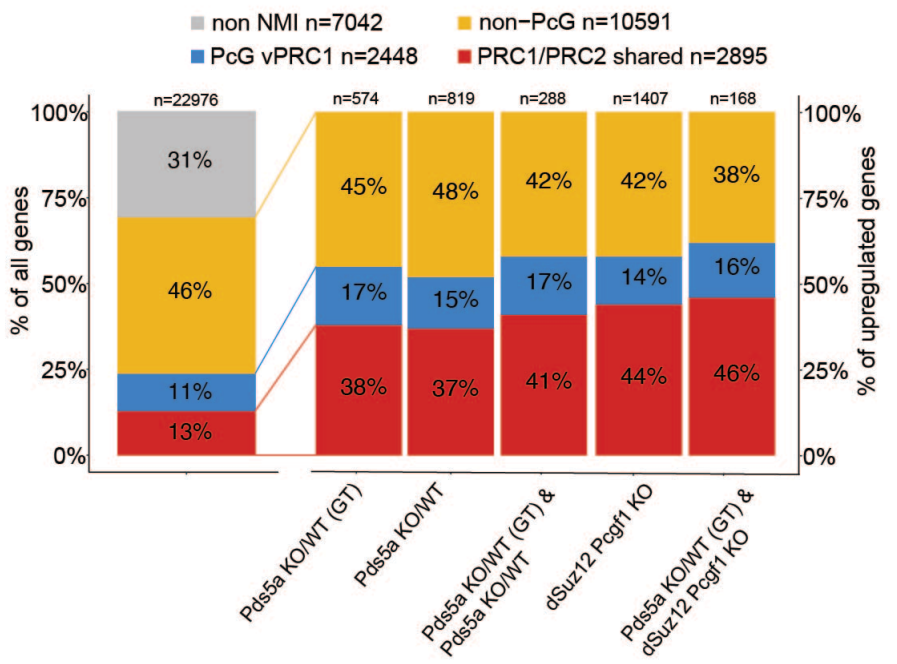

h

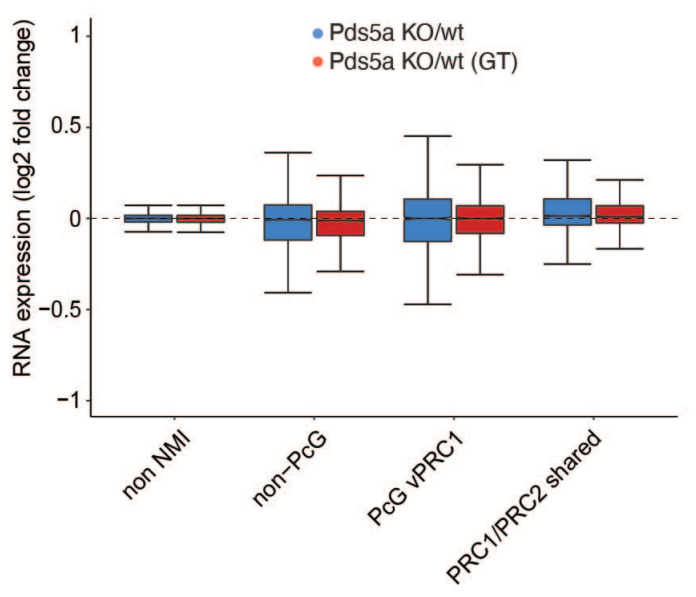

Bsteh et al., 
bioRxiv preprint doi: https://doi.org/10.1101/2021.12.15.472841; this version posted December 16, 2021. The copyright holder for this preprint (which was not certified by peer review) is the author/funder. All rights reserved. No reuse allowed without permission.

Fig. 3
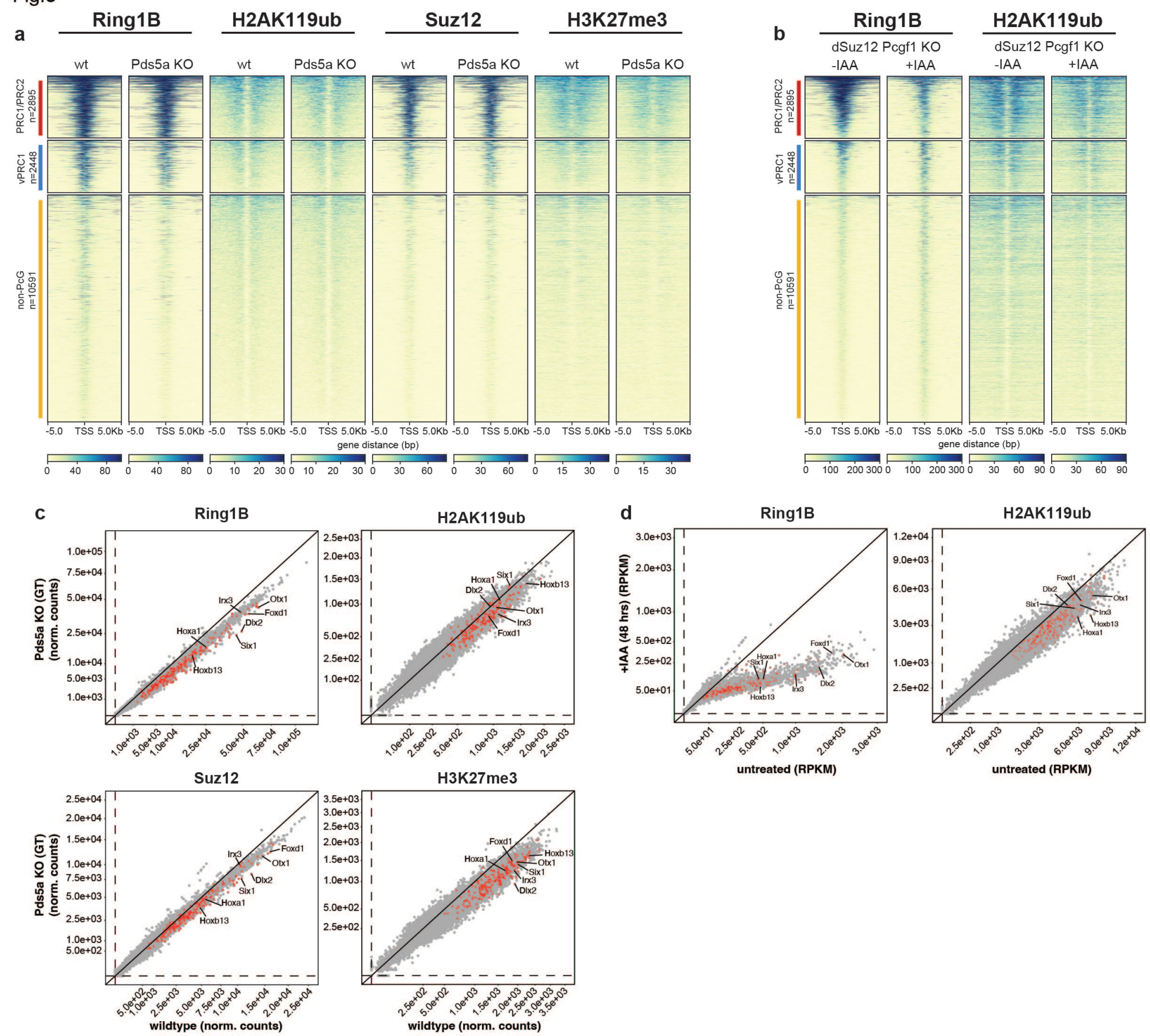

e

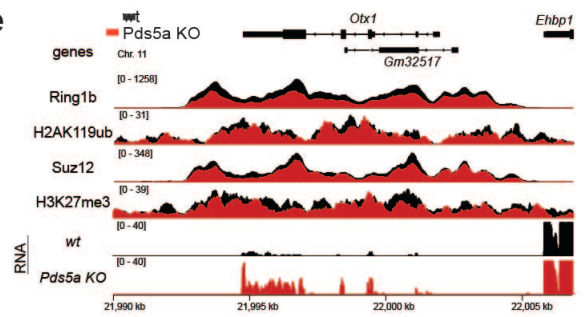

f

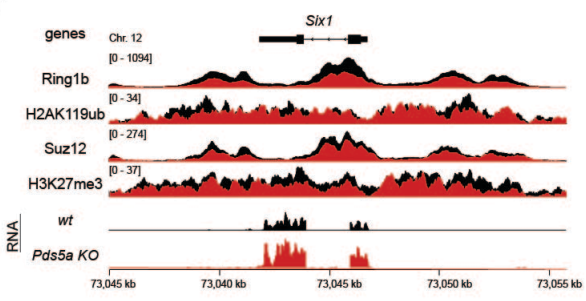

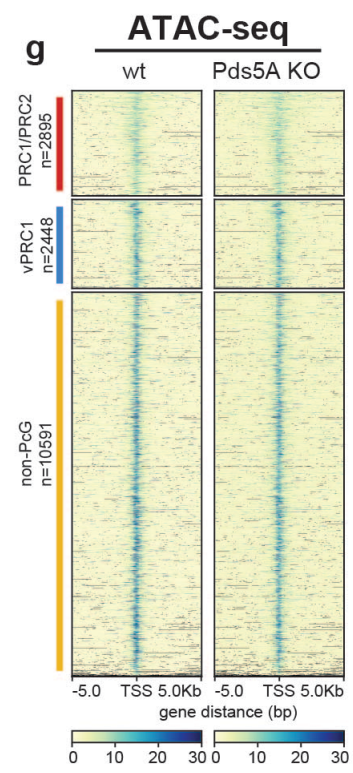

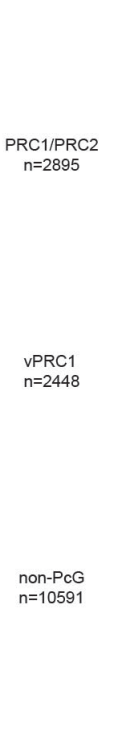

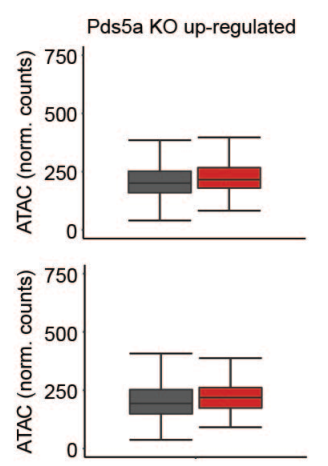

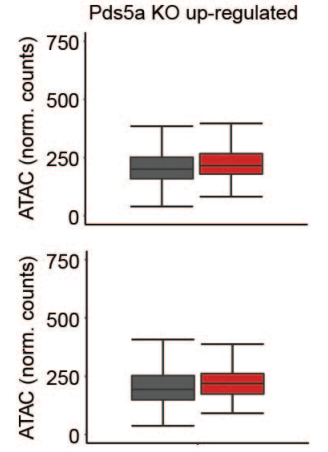

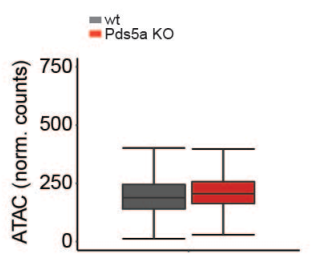
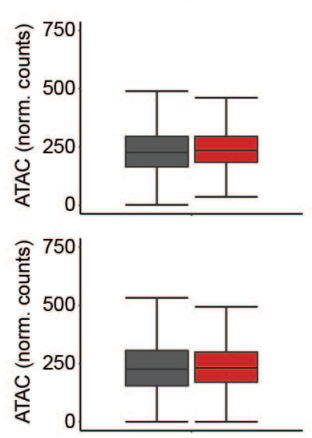
a

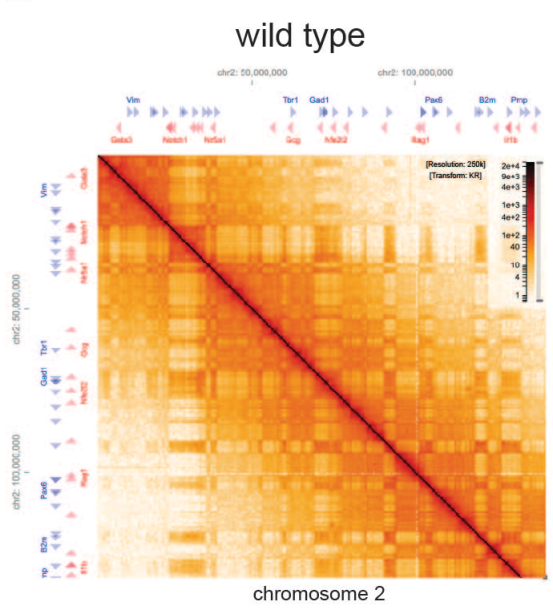

d

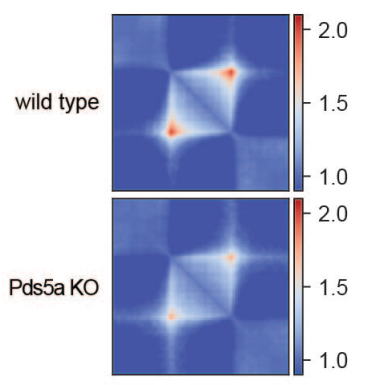

e

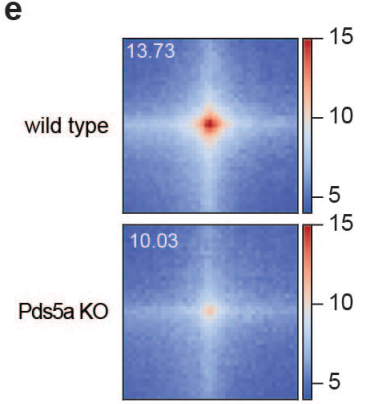

b

Pds5a KO
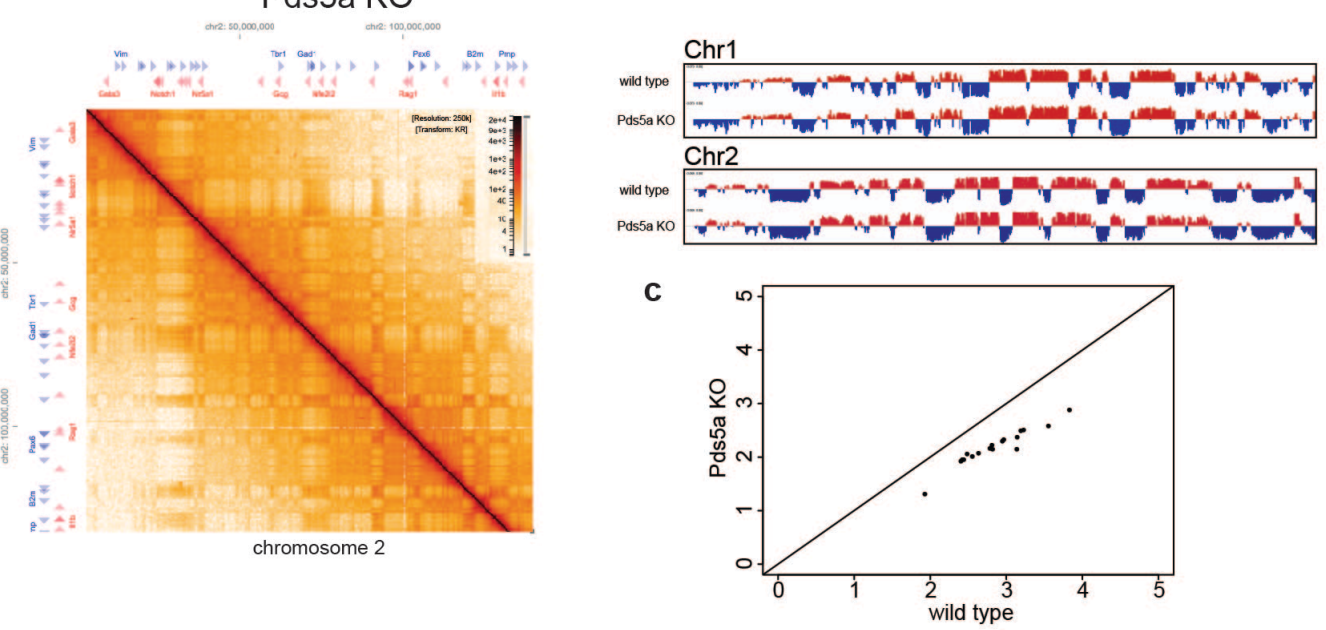

g
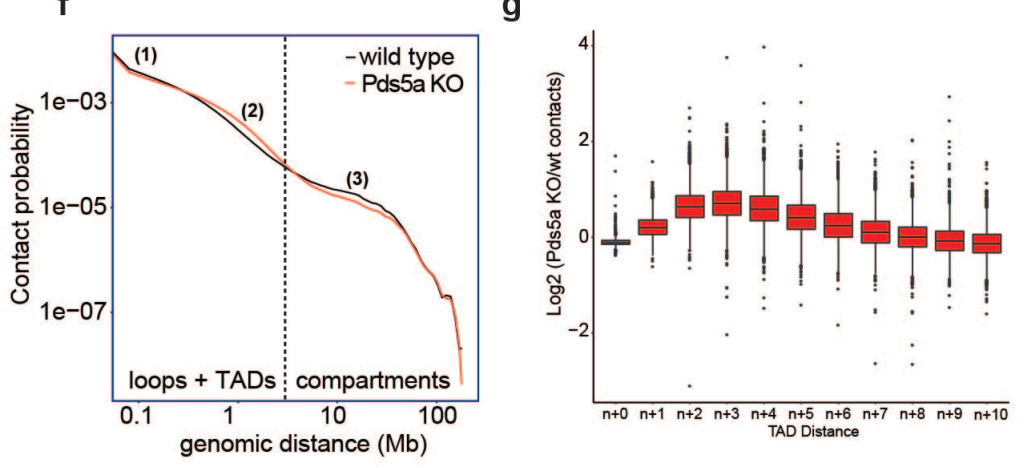
bioRxiv preprint doi: https://doi.org/10.1101/2021.12.15.472841; this version posted December 16, 2021. The copyright holder for this preprint (which was not certified by peer review) is the author/funder. All rights reserved. No reuse allowed without permission.

Fig.5

Bsteh et al.,

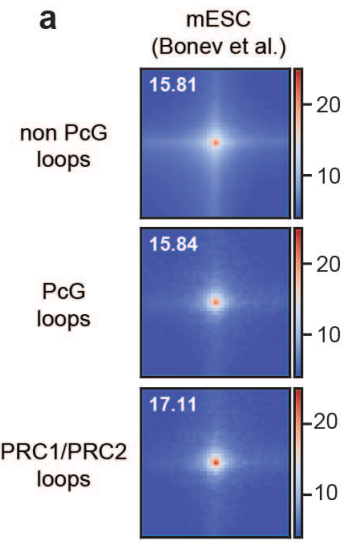

C

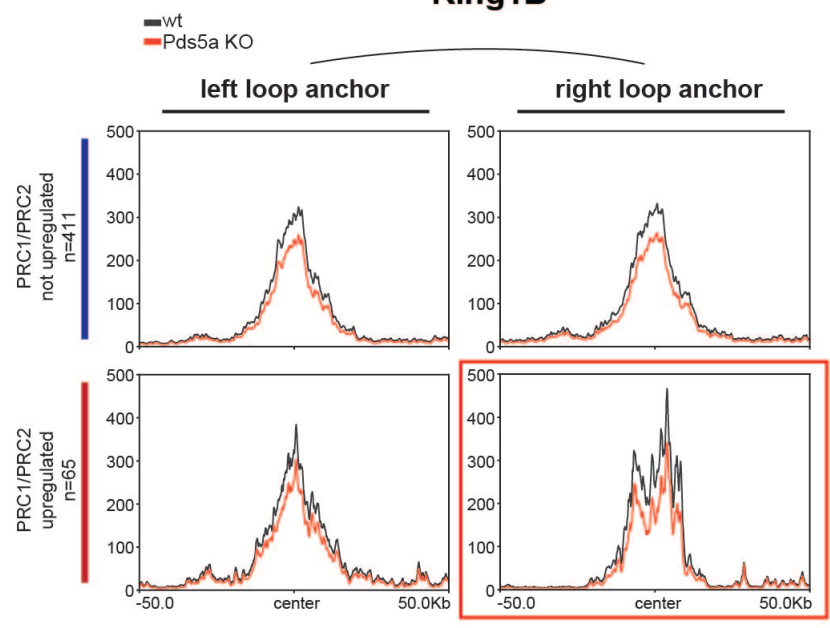

Suz12

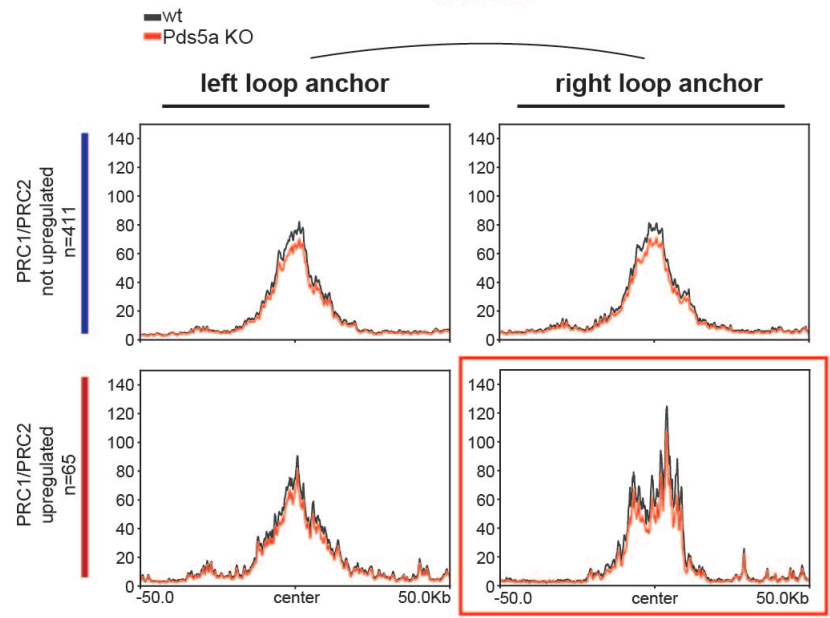

d
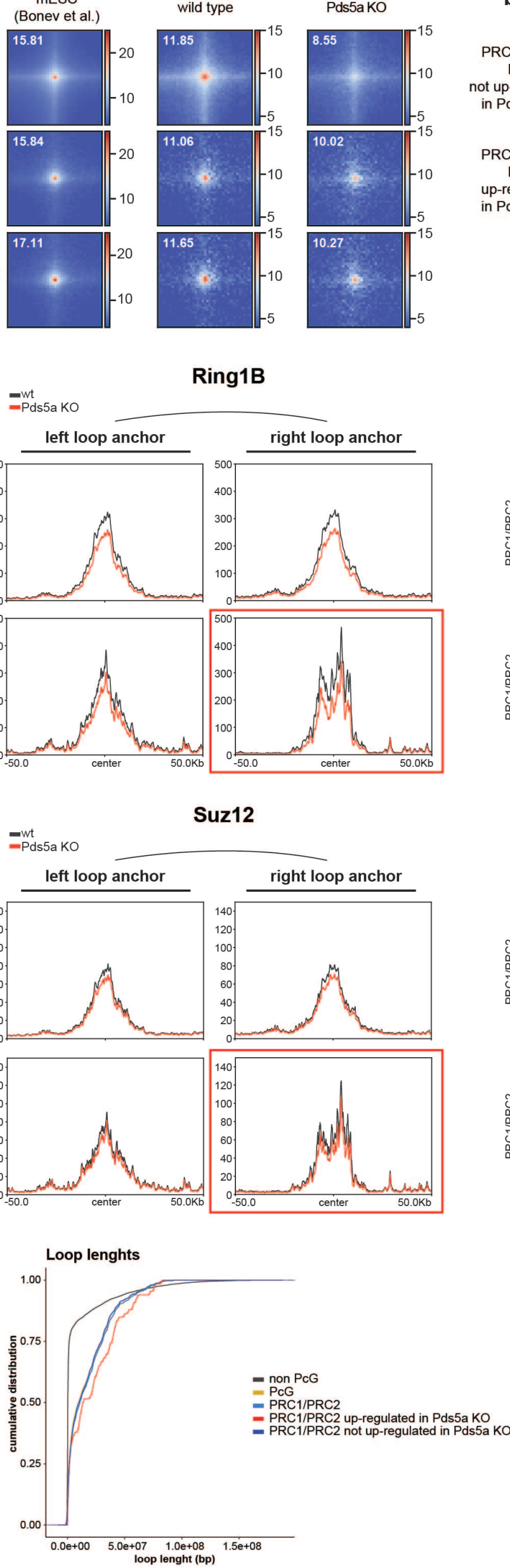

Ring1B b

PRC1/PRC2 loop not up-regulated in Pds5a KO

PRC1/PRC2

loop

up-regulated in Pds5a KO
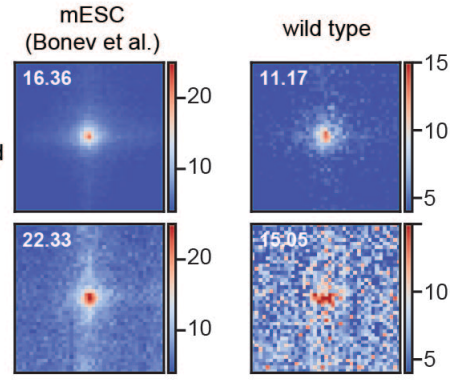

Pds5a KO

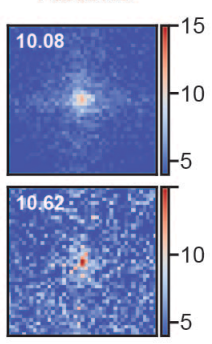

H2AK119ub

$=$ = Pds5a KO
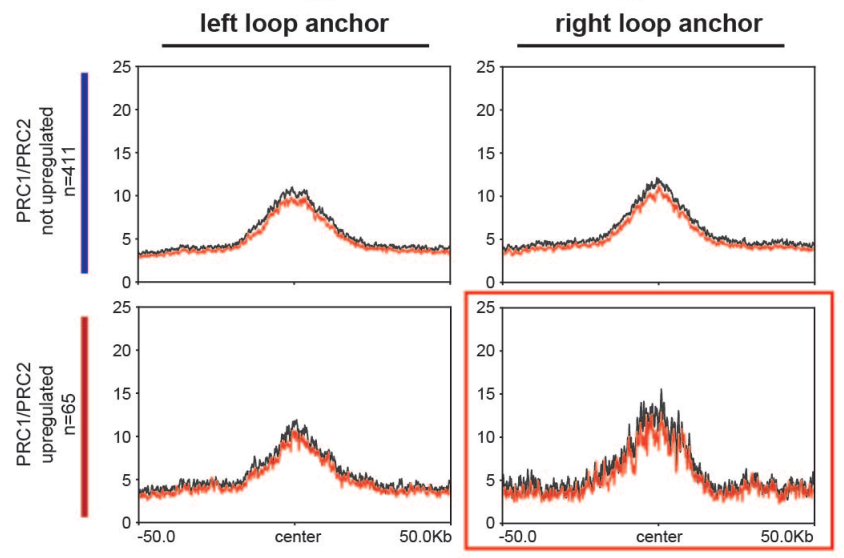

H3K27me3

$=$ Pds5a KO
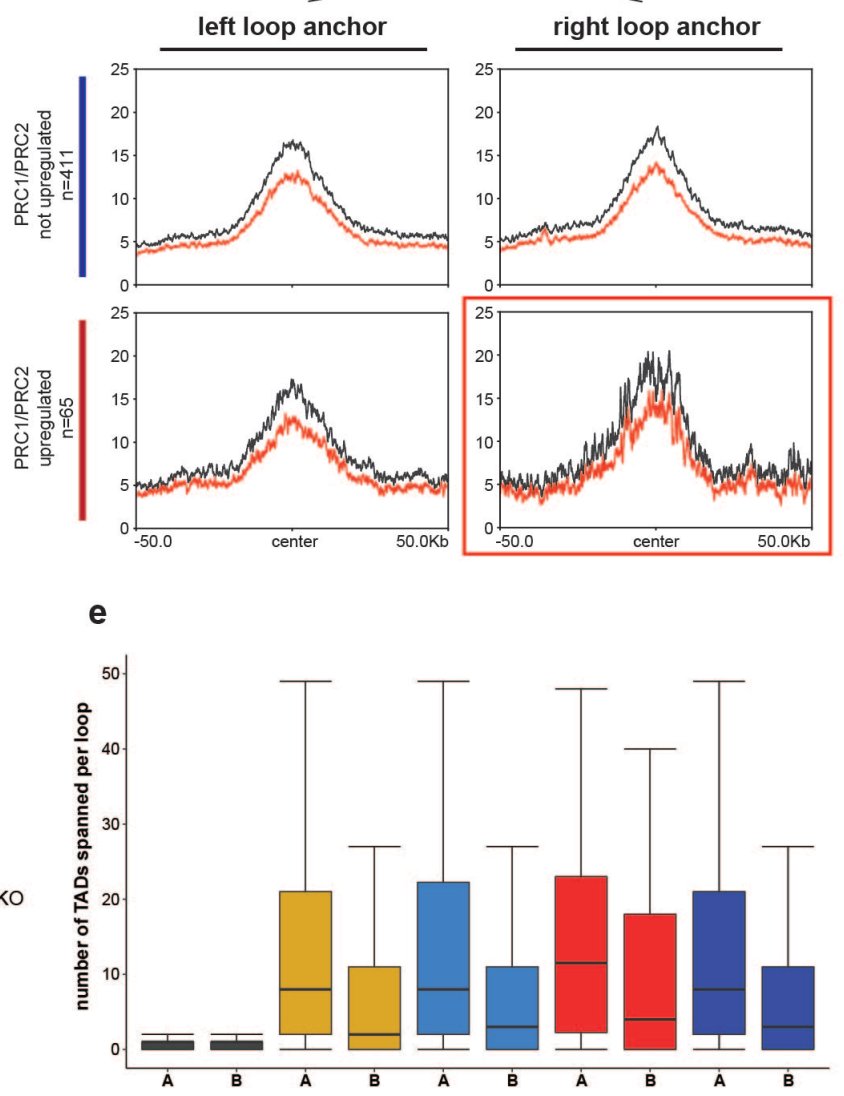
a

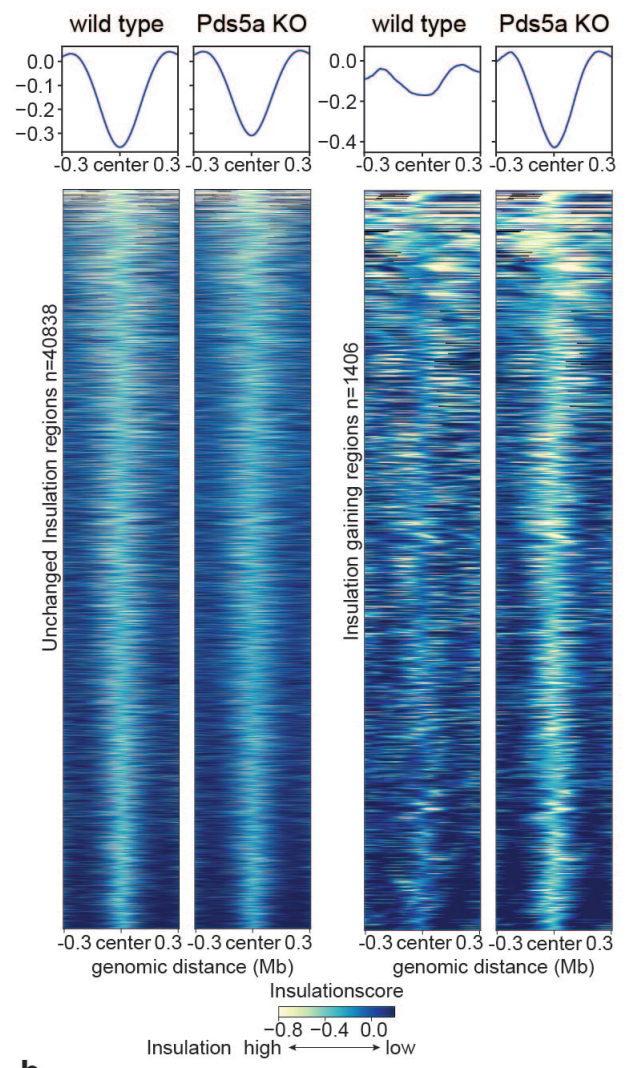

b

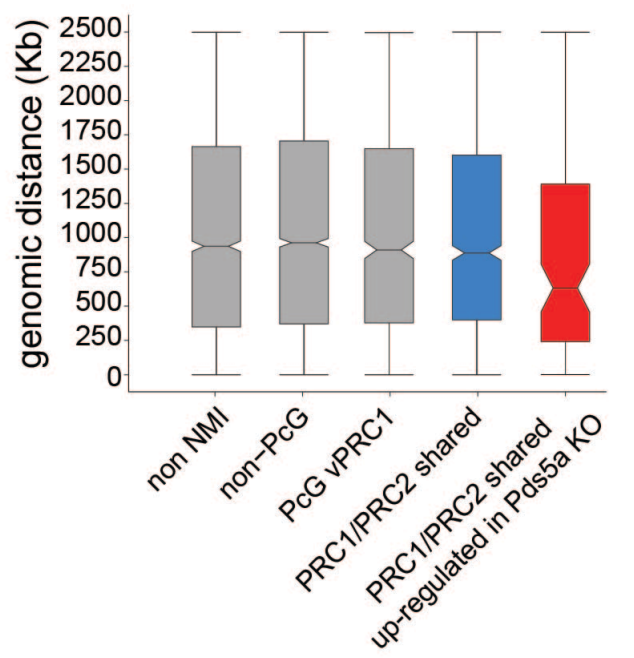

C

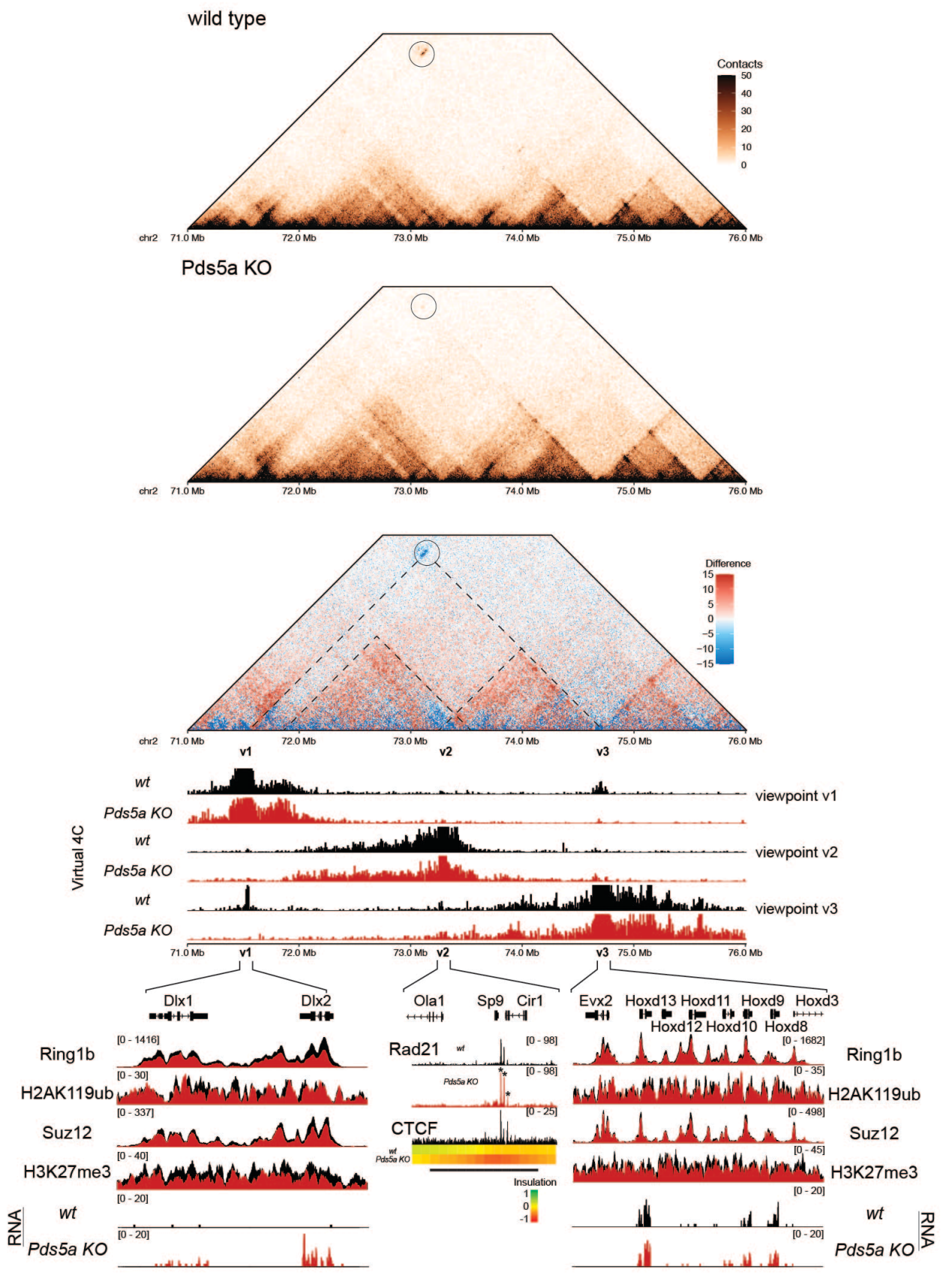


a

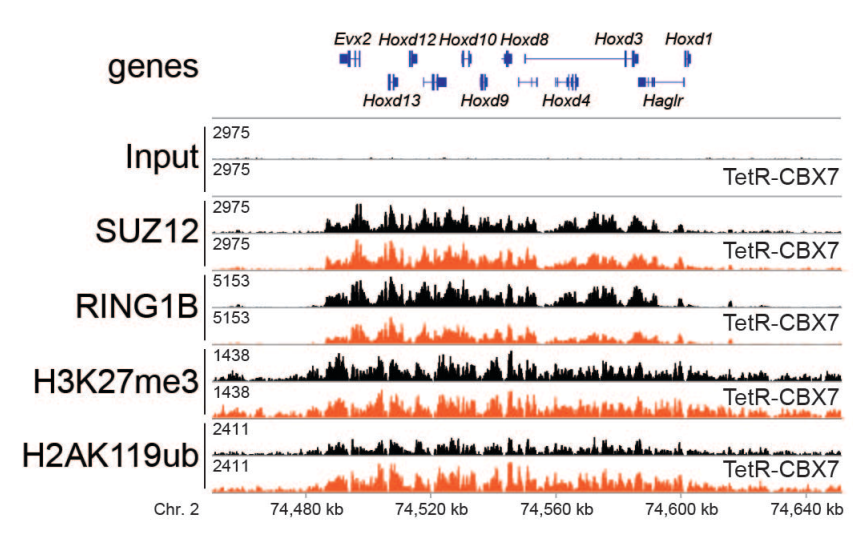

b
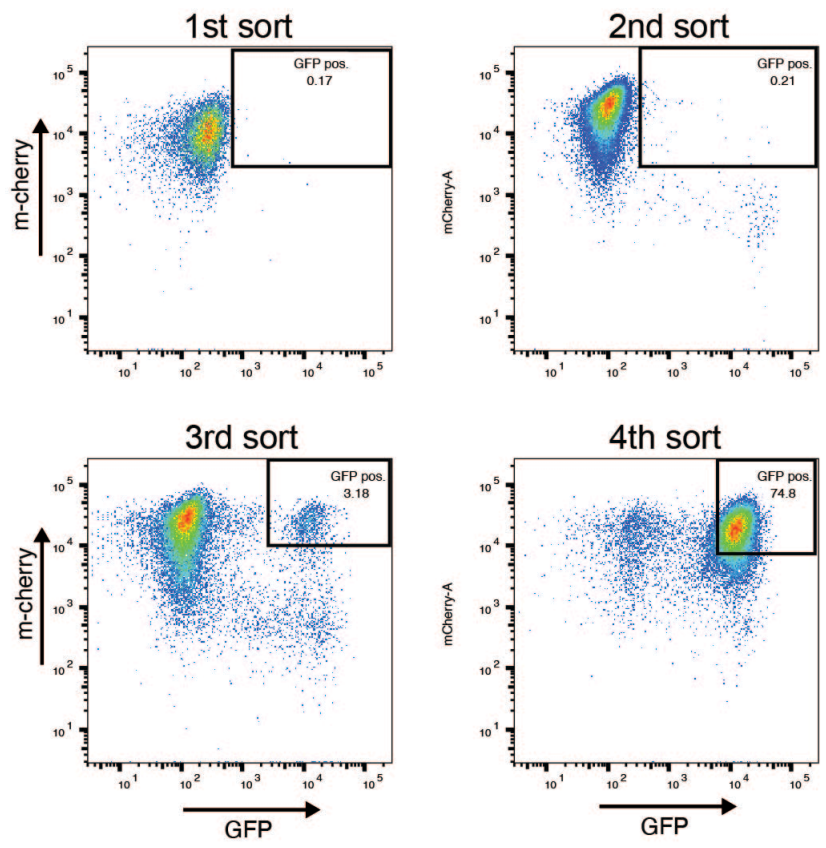

C
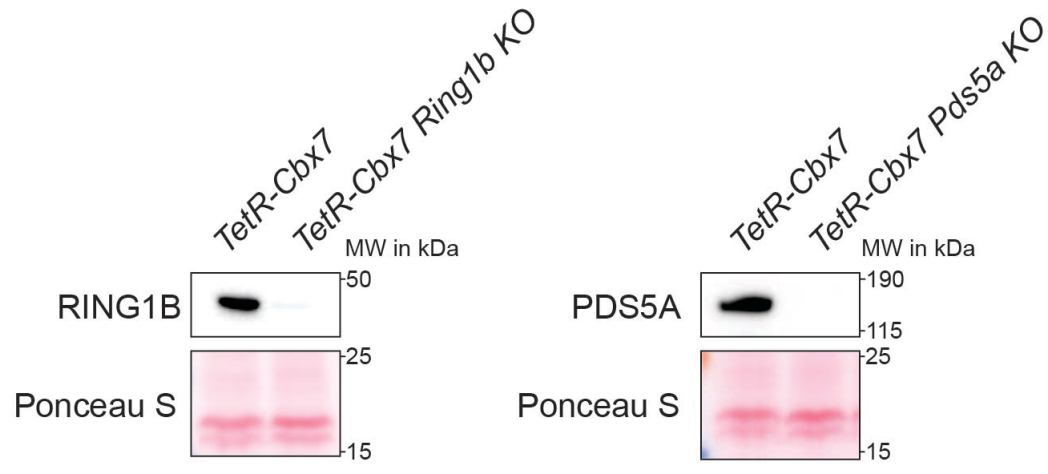
bioRxiv preprint doi: https://doi.org/10.1101/2021.12.15.472841; this version posted December 16, 2021. The copyright holder for this preprint (which was not certified by peer review) is the author/funder. All rights reserved. No reuse allowed without permission.

\section{Extended Data Fig.2}

Bsteh et al.,

a

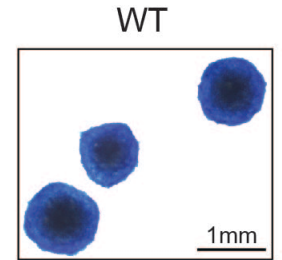

Pds5a KO

b

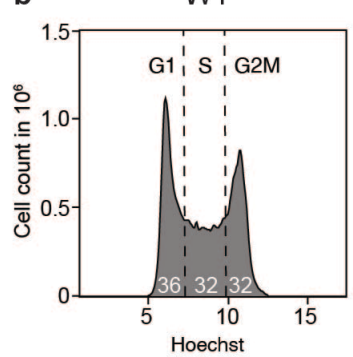

C
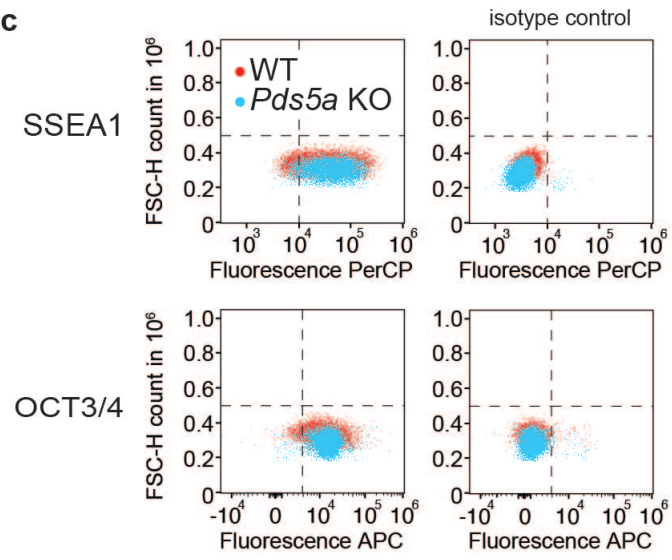

g
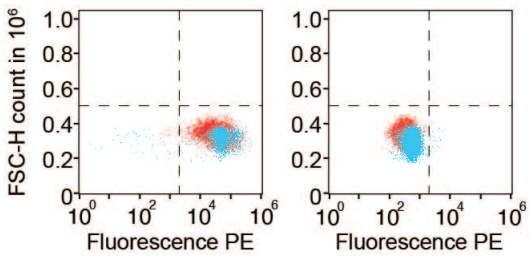

d

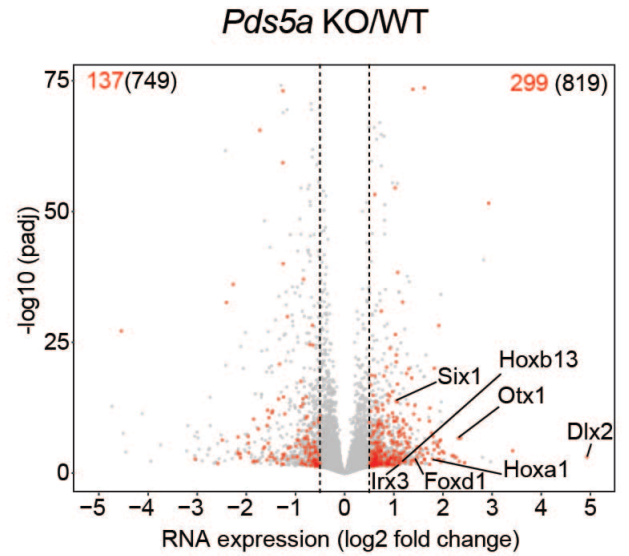

e

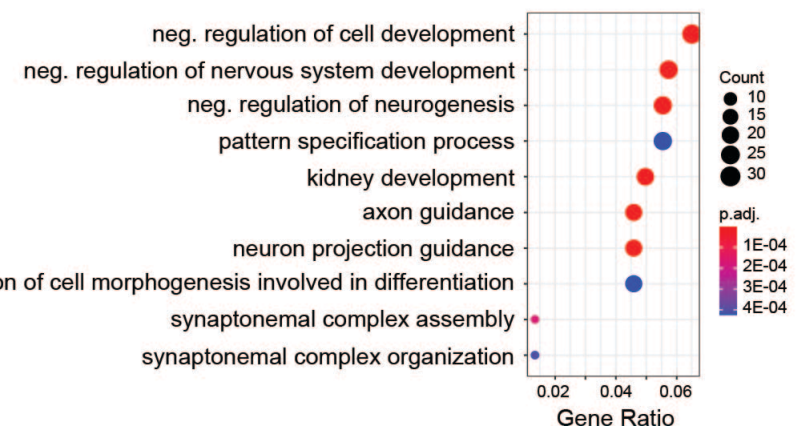

$\mathbf{f}$

Pds5a KO/WT

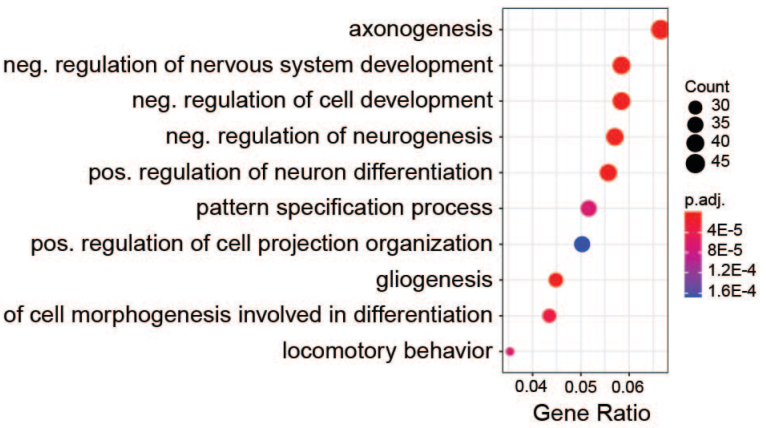

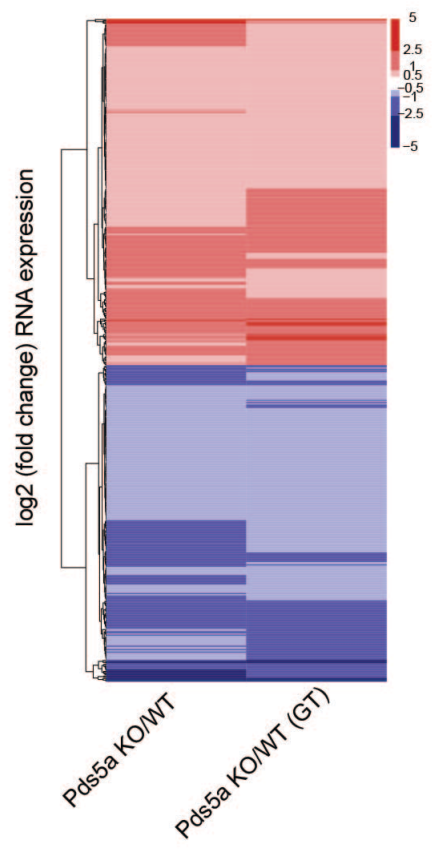

h

i
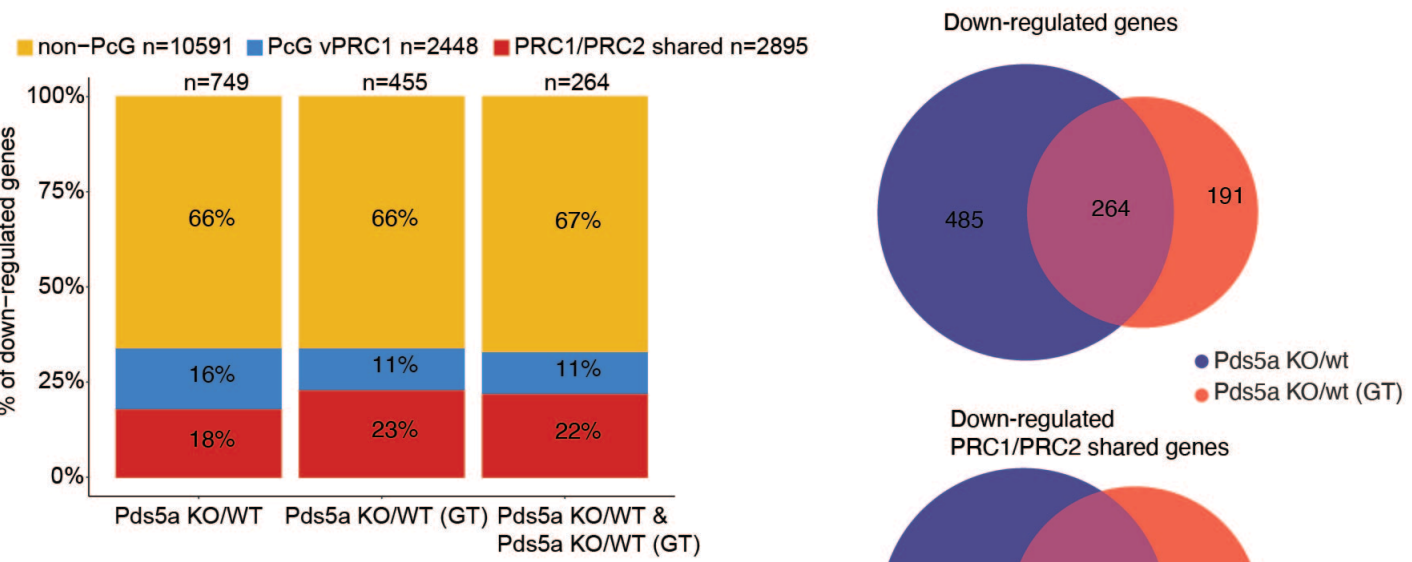

Down-regulated PRC1/PRC2 shared genes

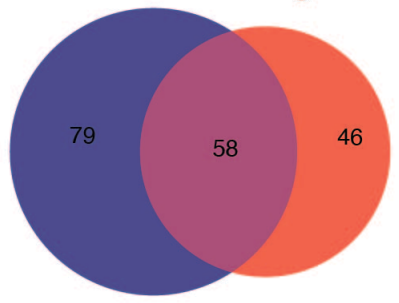




\section{Extended Data Fig.3}

a $\quad=$ =Pds5a KO

-PRC1/PRC2 shared $n=2895$-NMI PcG vPRC1 only $n=2448$ = NMI non-PcG $n=10591$
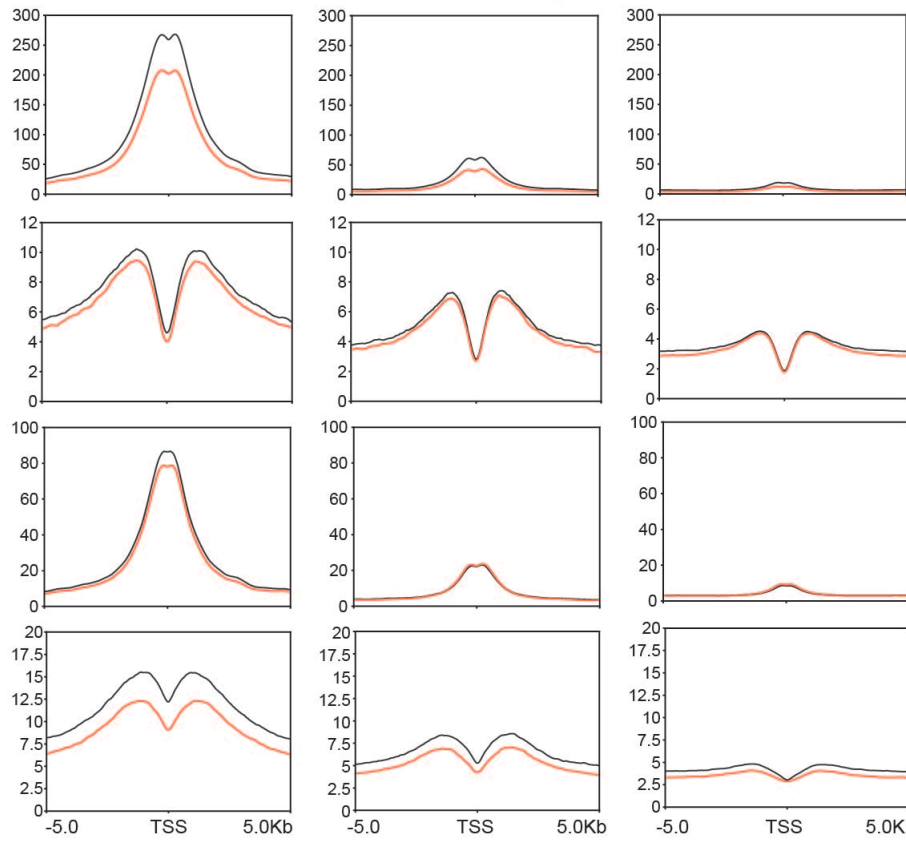

C
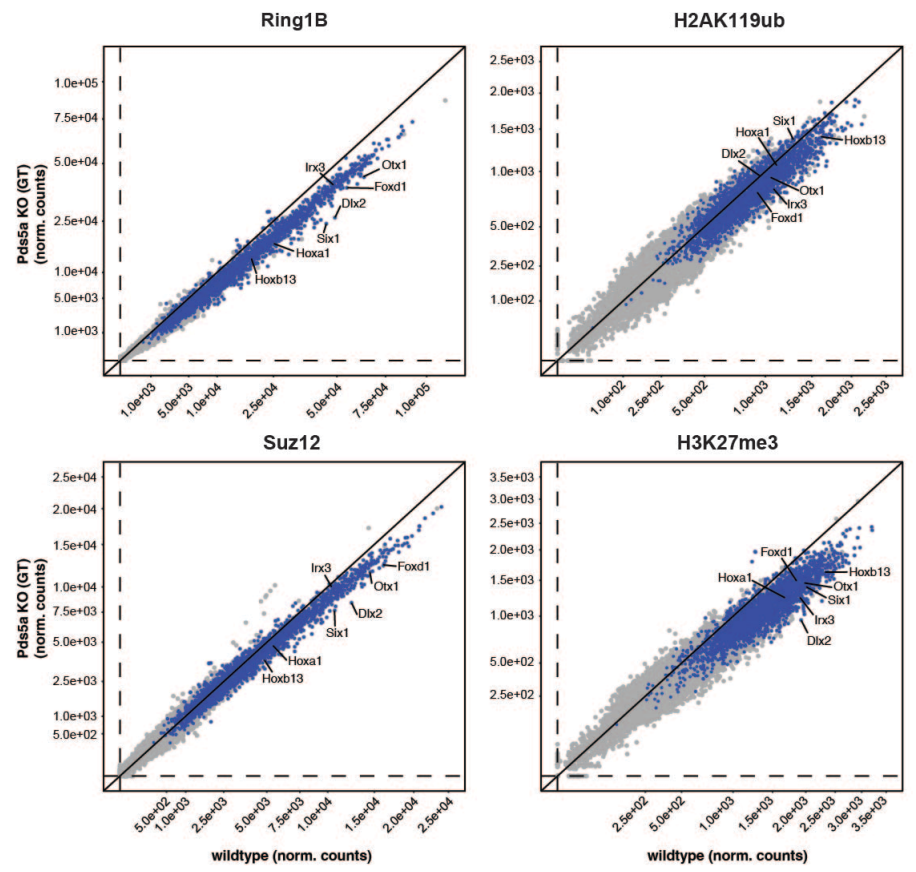

d
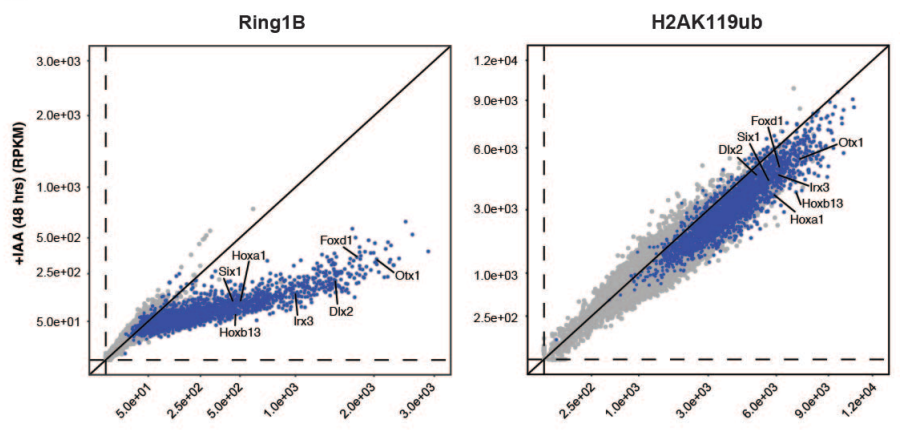
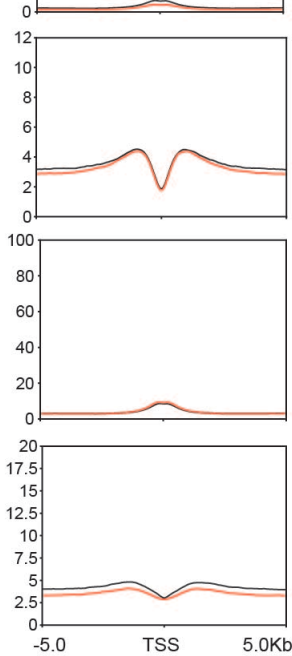

H3K27me3

H2AK119ub

Suz12 b

Ring1B

PRC1/PRC2 shared $n=2895=$ NMI PcG vPRC1 only $n=2448=$ NMI non-PcG $n=10591$
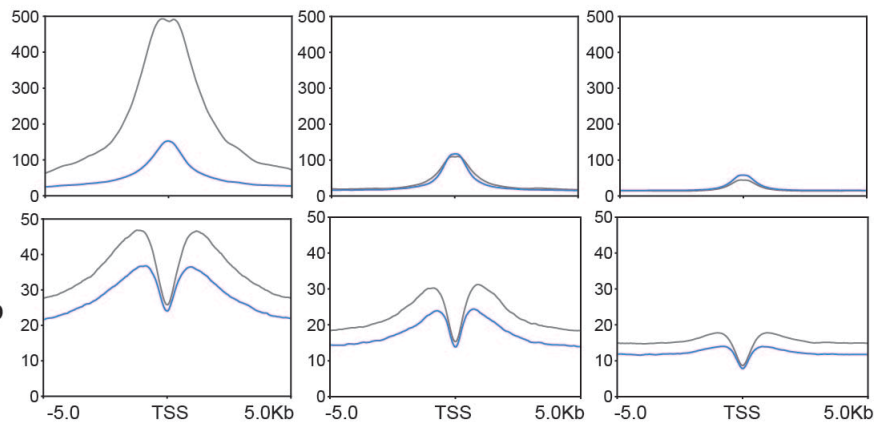

e
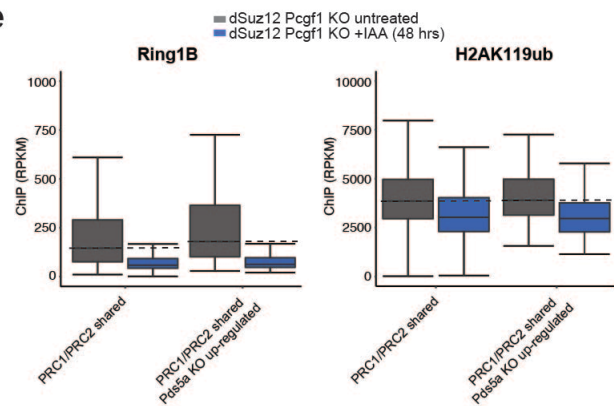

f
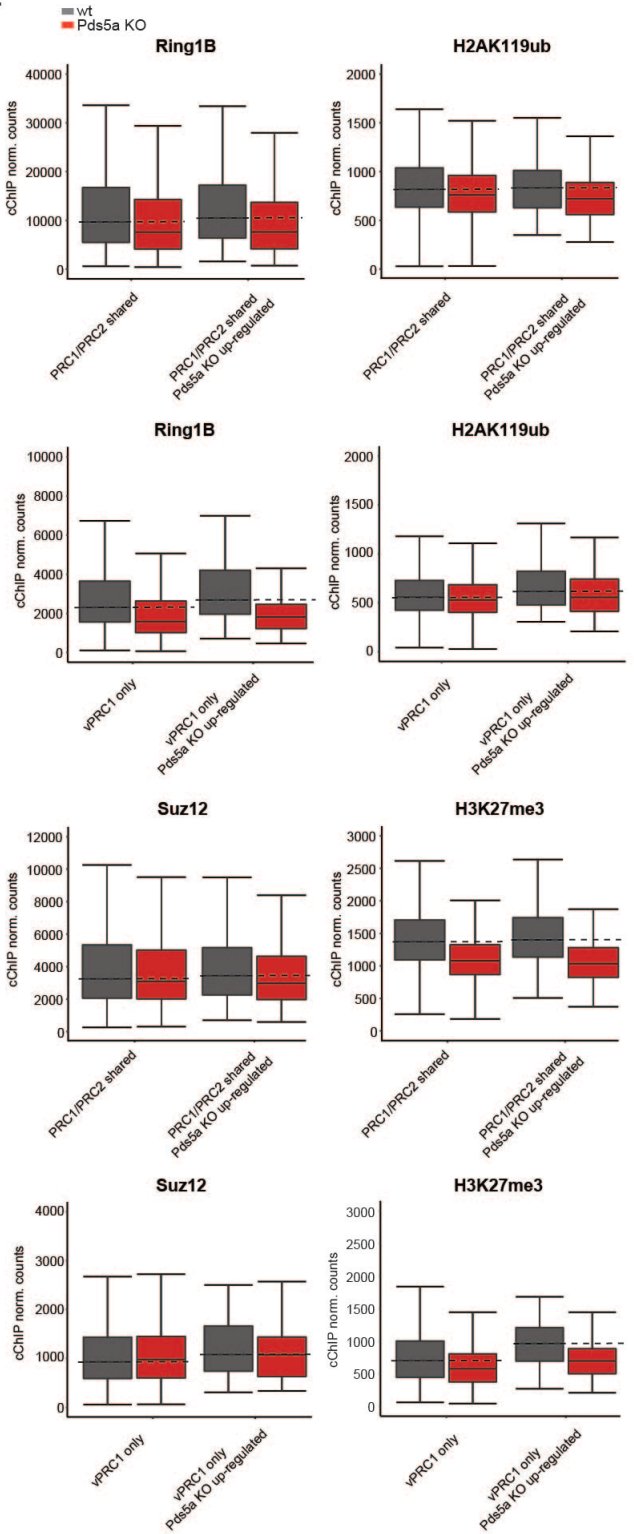
bioRxiv preprint doi: https://doi.org/10.1101/2021.12.15.472841; this version posted December 16, 2021. The copyright holder for this preprint (which was not certified by peer review) is the author/funder. All rights reserved. No reuse allowed without permission.

Extended Data Fig.4 a

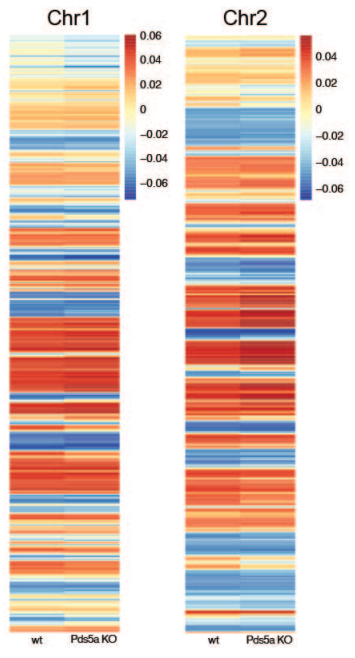

b

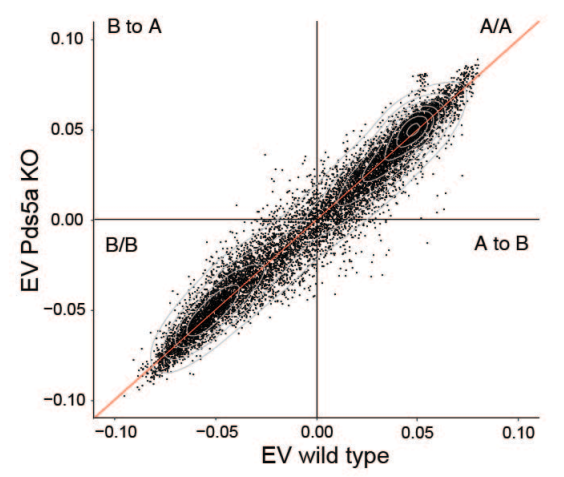

Bsteh et al.,

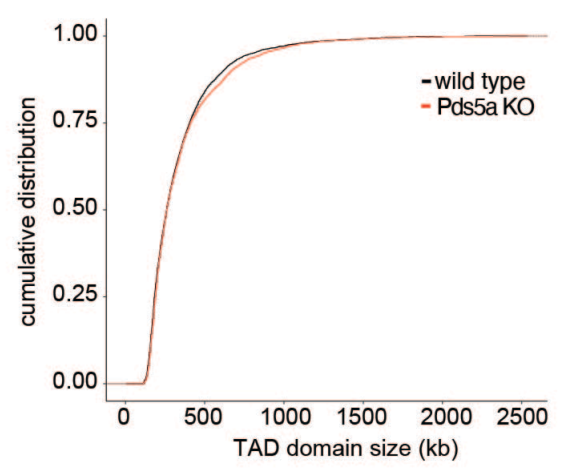

d

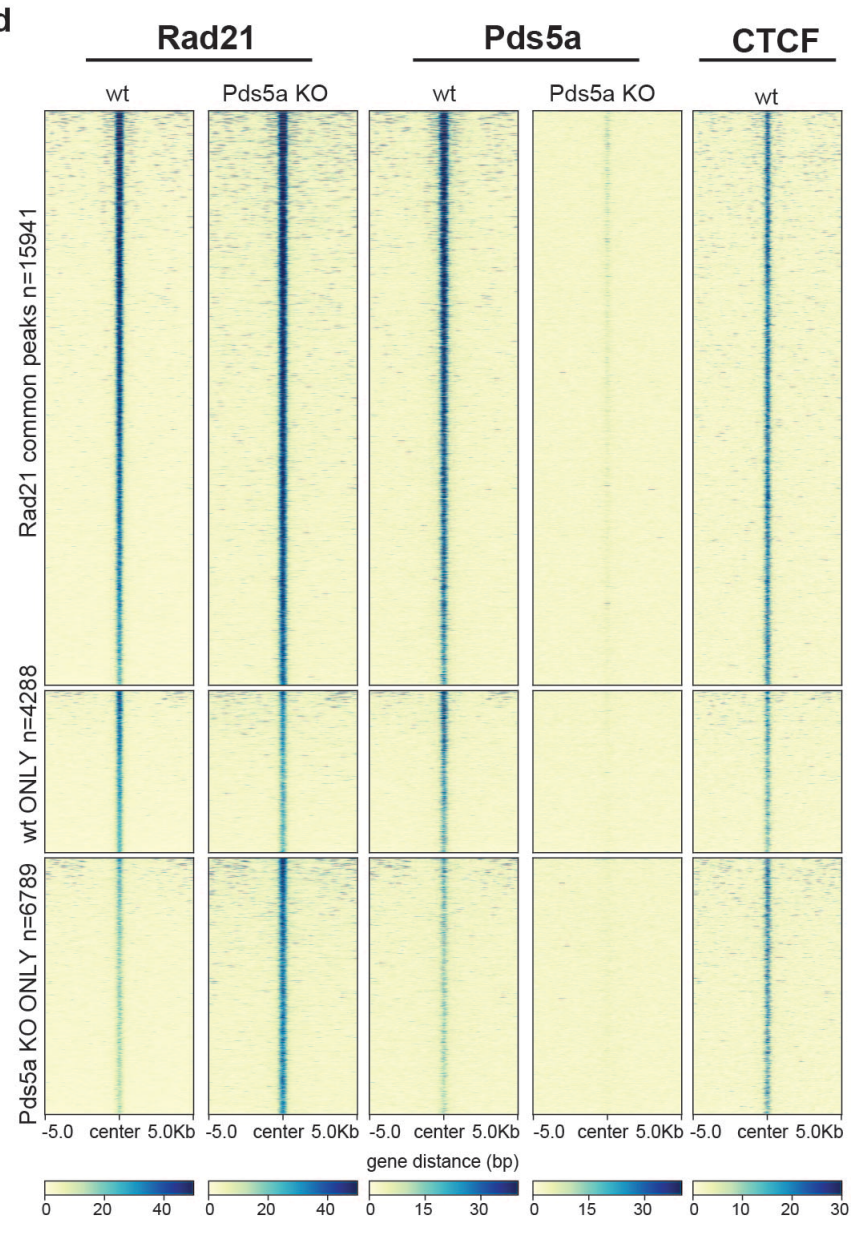

e

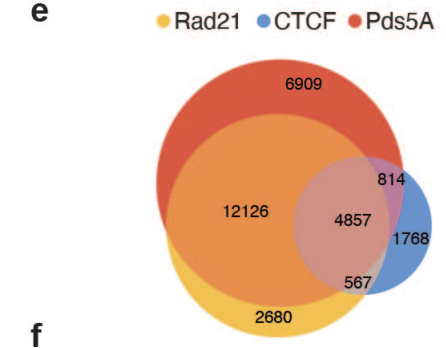

\section{f}

Rad21 common peaks $n=15941 \quad$ wt only $n=4288$

Pds5a KO only $n=6789$
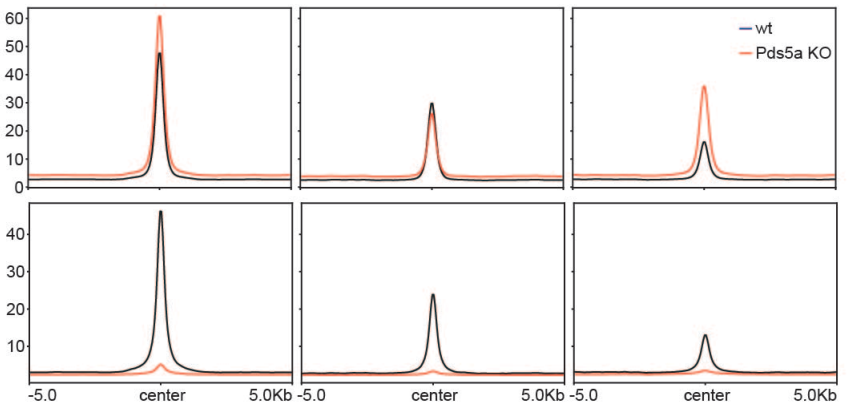

Pds5a

\section{g}

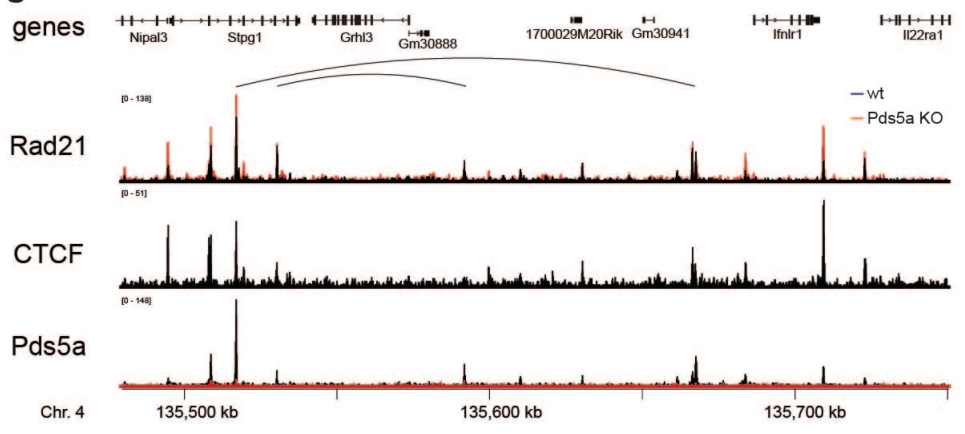


bioRxiv preprint doi: https://doi.org/10.1101/2021.12.15.472841; this version posted December 16, 2021. The copyright holder for this preprint (which was not certified by peer review) is the author/funder. All rights reserved. No reuse allowed without permission.

a

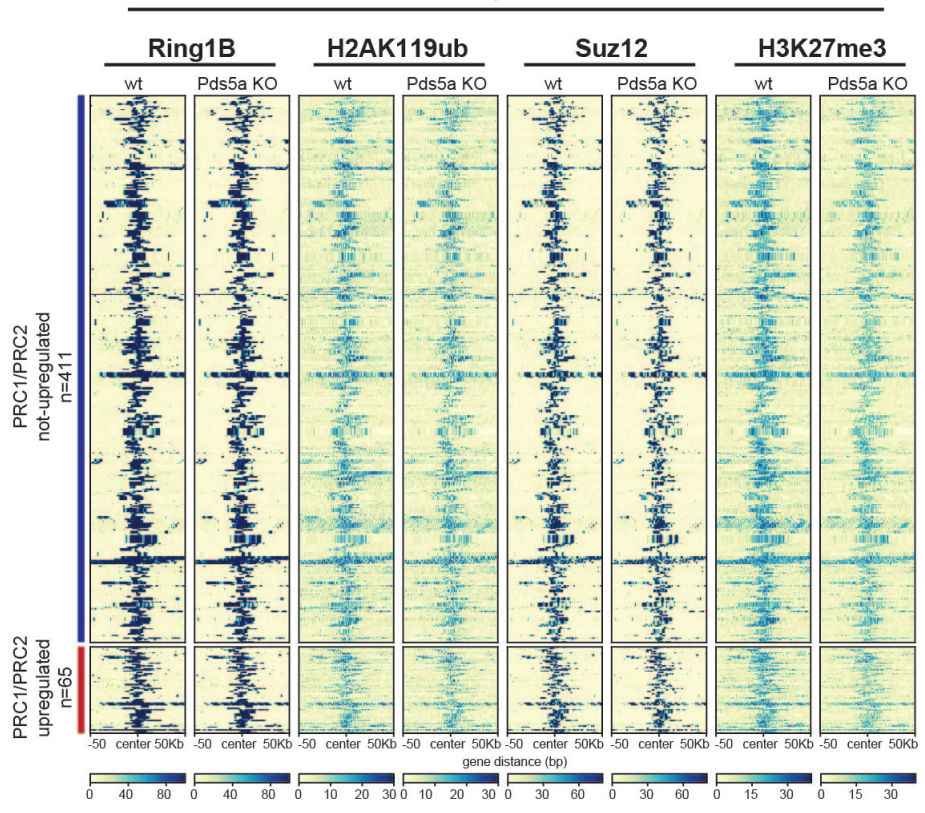

right loop anchor

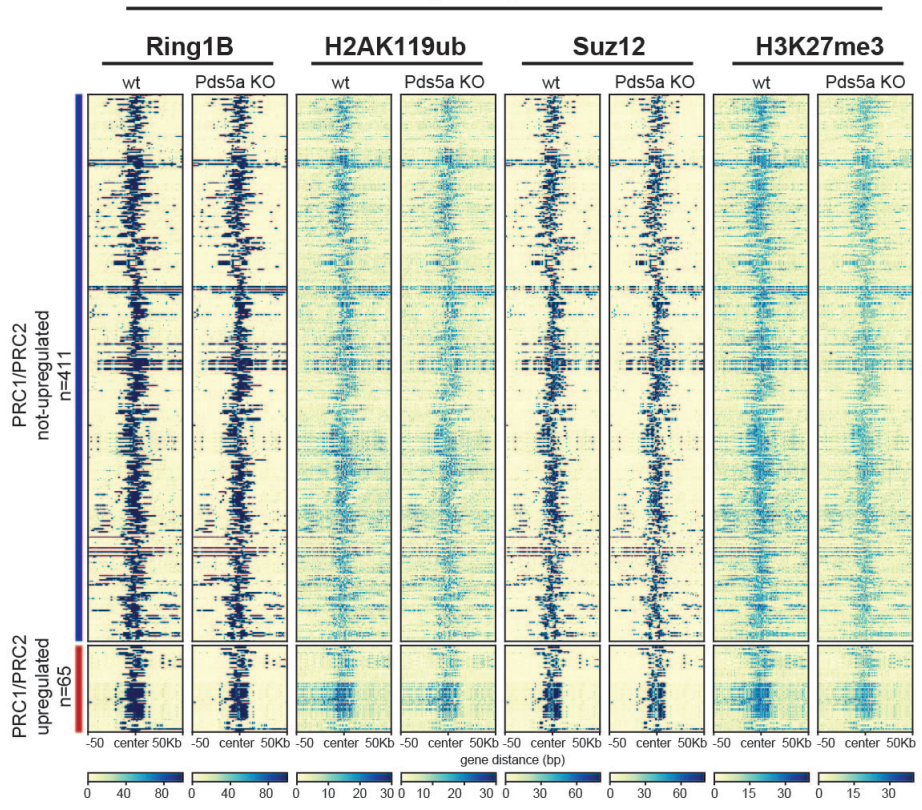


bioRxiv preprint doi: https://doi.org/10.1101/2021.12.15.472841; this version posted December 16, 2021. The copyright holder for this preprint (which was not certified by peer review) is the author/funder. All rights reserved. No reuse allowed without permission.

\section{Extended Data Fig.6}

a
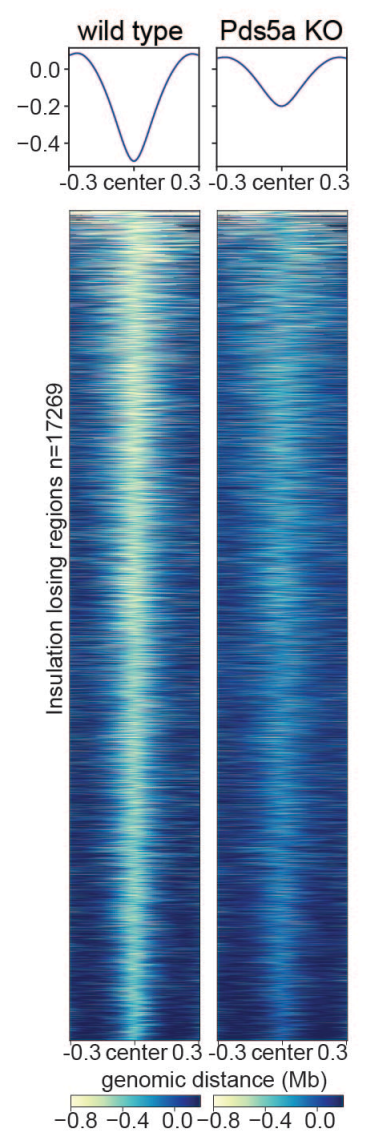

b

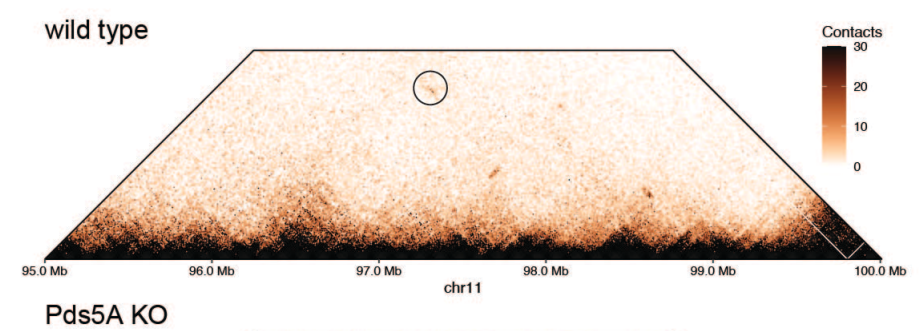

Suz12 H3K27me3
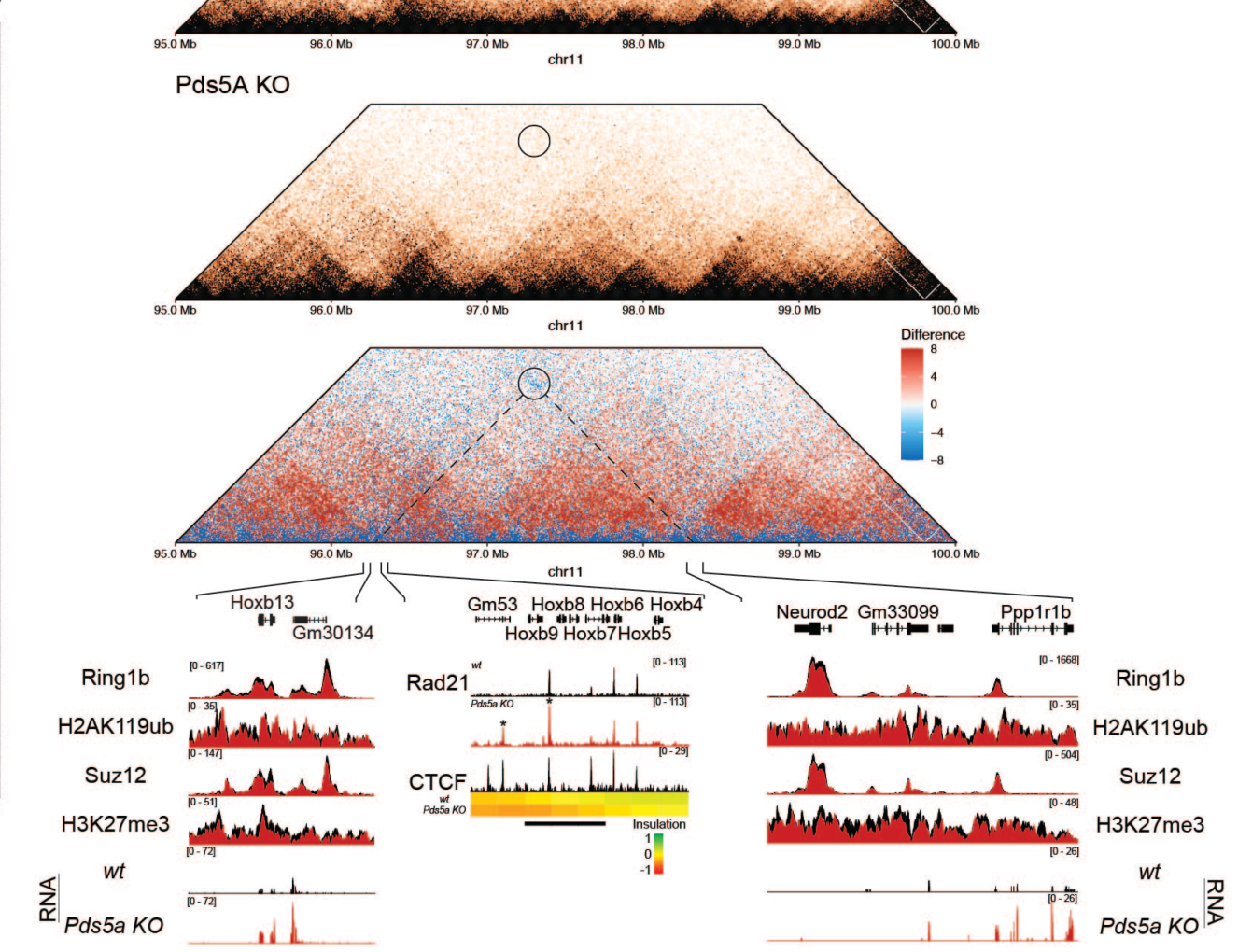

Bsteh et al., 